\title{
1 Respiratory complex and tissue lineage drive mutational patterns in the tumor 2 mitochondrial genome
}

Alexander N. Gorelick ${ }^{1,5}$, Minsoo Kim ${ }^{1}$, Walid K. Chatila ${ }^{1,4,5}$, Konnor La $^{2}$, A. Ari Hakimi ${ }^{3}$, Barry S. 5 Taylor ${ }^{1,4,5}$, Payam A. Gammage ${ }^{6,7^{*},}$, Ed Reznik ${ }^{1,4, *, \dagger}$

$6{ }^{1}$ Computational Oncology Service, Memorial Sloan Kettering Cancer Center

$7 \quad{ }^{2}$ Laboratory of Metabolic Regulation and Genetics, Rockefeller University

$8{ }^{3}$ Urology Service, Memorial Sloan Kettering Cancer Center

$9{ }^{4}$ Marie-Josee and Henry R. Kravis Center for Molecular Oncology, Memorial Sloan Kettering Cancer

10 Center

$11{ }^{5}$ Human Oncology and Pathogenesis Program, Memorial Sloan Kettering Cancer Center

$12{ }^{6}$ CRUK Beatson Institute, Glasgow, UK

$13{ }^{7}$ Institute of Cancer Sciences, University of Glasgow, Glasgow, UK

14 "Correspondence to: Ed Reznik (reznike@mskcc.org) and Payam Gammage

15 (payam.gammage@glasgow.ac.uk)

$16{ }^{\dagger}$ Lead Contact

\section{Abstract}

Mitochondrial DNA (mtDNA) encodes essential protein subunits and translational machinery for four distinct complexes of oxidative phosphorylation (OXPHOS). Using repurposed wholeexome sequencing data, we demonstrate that pathogenic mtDNA mutations arise in tumors at a rate comparable to the most common cancer driver genes. We identify OXPHOS complexes as critical determinants shaping somatic mtDNA mutation patterns across tumor lineages. Loss-offunction mutations accumulate at an elevated rate specifically in Complex I, and often arise at specific homopolymeric hotspots. In contrast, Complex $\mathrm{V}$ is depleted of all non-synonymous mutations, suggesting that mutations directly impacting ATP synthesis are under negative selection. Both common truncating mutations and rarer missense alleles are associated with a pan-lineage transcriptional program, even in cancer types where mtDNA mutations are comparatively rare. Pathogenic mutations of mtDNA are associated with substantial increases in overall survival of colorectal adenocarcinoma patients, demonstrating a clear functional relationship between genotype and phenotype. The mitochondrial genome is therefore frequently and functionally disrupted across many cancers, with significant implications for 


\section{Introduction}

40 Somatic mutations are the underlying drivers of malignancy in cancer, and the identification and

41 characterization of recurrent, functional somatic events has been the capstone goal of cancer

42 genomics. Genomic searches for recurrent driver mutations have focused on the nuclear exome

43 or subsets thereof, motivated by the observation that recurrent mutations are concentrated in

44 the coding regions of a subset of nuclear-DNA-encoded genes. This targeted approach has

45 powered the discovery of common and rare driver mutations in exonic regions, but by corollary

46 has also left underexplored the overwhelming majority of the genome and the driver events it

47 may harbor. Numerous examples now exist of the prevalence and function of oncogenic

48 mutations beyond the nuclear exome, including mutations to the TERT promoter, non-coding

49 RNAs including ribosomal RNA and snRNAs, and enhancers ${ }^{1}$. A fundamental challenge is

50 therefore to discover new functional somatic alterations beyond the exome with a fixed and

51 limited sequencing capacity.

Somatic mutations in tumors commonly target human mitochondrial DNA (mtDNA) ${ }^{2-6}$, affecting

54 both the thirteen essential protein components of four distinct complexes ( $\mathrm{Cl}, \mathrm{CIII}$, CIV, and CV)

55 in oxidative phosphorylation (OXPHOS) as well as the non-coding RNA (22 tRNAs, 2 rRNAs) necessary for mtDNA translation. (Fig. 1a). Despite abundant pharmacological, genetic, and

57 clinical data demonstrating that perturbation of different OXPHOS complexes (referred to in shorthand as complexes) produce distinct cellular adaptations ${ }^{7,8}$, the importance of each complex in shaping mtDNA mutation patterns in cancer is unknown. Because mtDNA is not commonly targeted by exome sequencing panels, prior analyses of mtDNA mutations have relied on cohorts profiled with whole genome sequencing, with consequently diminished statistical power to detect recurrent patterns of mutations relative to exome sequencing studies ${ }^{8}$.

63 However, due to the extremely high copy number and off-target hybridization rate of mtDNA,

64 mtDNA reads are abundant in widely-available exome sequencing of tumors ${ }^{9}$. Mitochondrial

65 DNA therefore represents an opportunity for discovery through repurposing of existing exome 66 sequencing data.

Here, by utilizing existing exome sequencing data to more than double statistical power of prior analyses, we report that OXPHOS complex, in combination with tissue lineage and mutational consequence, is a critical determinant of mtDNA mutation patterns in cancer. We find that

$71 \mathrm{NADH}$ :ubiquinone oxidoreductase (complex I, $\mathrm{Cl}$ ) mutations are strongly enriched for highly

72 pathogenic mutations in specific tissue lineages, whereas ATP synthase (complex $V, C V$ ) is 
73 broadly depleted of all non-synonymous mutations. We further identify six highly recurrent

74 mtDNA mutation hotspots at specific homopolymer sequence contexts, which collectively

75 account for over $40 \%$ of all truncating mutations to mtDNA, as well as recurrent mutations in

76 both protein-coding genes and non-coding RNA elements. These mutations produce a defined,

77 lineage-agnostic transcriptional program and, in specific tumor lineages, associate with both

78 underlying molecular subtypes and clinical outcomes. Our results argue that specific

79 components of mitochondrial respiration are broadly perturbed across many tissue lineages,

80 and that re-analysis of existing genomic data can yield new discoveries in underexplored

81 genomic terrain.

\section{Results}

\section{4 mtDNA Mutations in Tumors from Off-target Reads}

85 To study patterns of mtDNA mutations in tumors, we reasoned that the sheer amount of offtarget reads aligning to mtDNA in whole-exome sequencing data would be sufficient to call somatic mtDNA mutations in a large proportion of samples. We therefore assembled a dataset of pan-cancer paired tumor and matched-normal exome sequencing samples from the TCGA, $n=10,132$ (Supplementary Fig. 1a). Inconsistent sequencing coverage between samples is an inherent limitation to this approach, as variants located in regions without adequate sequencing coverage are not identifiable, and we therefore developed our methodology to be cognizant of the sequencing coverage at each position in each sample (see Methods). We focused our analysis on regions of mtDNA in protein-coding genes and genes coding for mitochondrial ribosomal RNAs (rRNAs) and transfer RNAs (tRNAs), excluding the control region (pos 1-576, 16,024-16,569), several known hypervariable loci (pos 302-314; 514-524; 3,106-3,109), and 89 remaining positions not within any genic region from all further analyses (excluded positions listed in SI table 1). The combination of an increase in sample size (in TCGA, relative to whole genome cohorts) and high off-target read coverage effectively doubled the number of tumorassociated mtDNA genomes sequenced compared to the largest published dataset of wholegenome sequenced tumor mitochondrial genomes ${ }^{3}$ : On average 6,100 tumors were sequenced at sufficient depth to call mutations at each mtDNA position (mean+/-SD: 5,399-6,800 samples covered at a given position, Fig. 1b, Supplementary Fig. 1b), compared to 2,836 wholegenome tumor sequences from the PCAWG dataset. When further restricted to regions

104 sequenced at sufficient depth in both tumor and matched-normal samples, each position was 105 covered in 4,769 tumor/normal pairs on average (mean+/-SD: 4,148-5,390 samples). 
We implemented a conservative variant calling approach modeled after state-of-the-art methodologies for exome sequencing, in which we took the intersection of two variant callers (MuTect2 ${ }^{10}$ and an in-house variant caller based on the SAMtools mpileup utility ${ }^{11}$, see Methods). Consistent with prior work, mtDNA variants exhibited a strand-specific enrichment for $\mathrm{C}>\mathrm{T}$ mutations on the heavy strand and $\mathrm{T}>\mathrm{C}$ mutations on the light strand (Supplementary

112 Fig. 1c). Based on 789 tumor samples from TCGA with whole genome sequences in the 113 PCAWG cohort ${ }^{3}, 95.6 \%$ of mutation calls from whole exome sequencing validated against 114 published mutation calls from the PCAWG data (Fig. 1c). We also evaluated the possibility that 115 nuclear-encoded mitochondrial pseudogenes (NUMTs) could corrupt variant calling. Although 116 both mtDNA and NUMTs are not targeted by exome sequencing, mtDNA is unique in that it exists at orders of magnitude higher copy number in each cell and, critically, is expressed at extremely high levels, whereas NUMTs do not show evidence of significant transcription ${ }^{12}$. We therefore determined the fraction of somatic mtDNA variants from exome sequencing which could be recapitulated in matched RNA sequencing from the same sample, revealing that $96.9 \%$ of such variants were validated. Finally, we observed a strong correlation between DNA and RNA heteroplasmy overall (Pearson's $r=0.918)$ (Fig. 1d), confirming that the vast majority of observed mutations are expressed and providing further evidence that the mutations called by our approach are not attributable to NUMTs. In total, we identified 4,381 mtDNA mutations from 10,132 tumor samples which were either protein-truncating (i.e. frame-shift indels or nonsense mutations); or non-truncating variants (missense, in-frame indels, translation startsite, non-stop, or mutations to tRNA/rRNAs) which were detected in tumor and absent from matched-normal samples. Among a subset of 3,264 paired tumor/normal samples with sufficient coverage to call mtDNA mutations in at least $90 \%$ of the mitochondrial genome $(32 \%$ of tumor/normal pairs in our dataset overall, referred to throughout as "well-covered" samples), $57 \%(95 \% \mathrm{Cl} 56-59 \%)$ had at least one mtDNA variant, in agreement with previous estimates for mtDNA mutation incidence in pan-cancer sequencing data ${ }^{2}$. Consistent with independent mutagenic processes operating in the nuclear and mitochondrial genomes, we observed no correlation between nuclear and mitochondrial mutation burdens pan-cancer or within individual cancer types (Fig. 1e, Supplementary Fig. 1d). Furthermore, in colorectal and stomach cancers where microsatellite instability $(\mathrm{MSI})$ is common, the presence of $\mathrm{MSI}$ affected mutation

137 burden in the nuclear but not in the mitochondrial genome (Supplementary Fig. 1e). than the rate in 468 cancer-associated genes in the MSK-IMPACT panel ${ }^{13}$ of 11.3 mut/Mb $(P<$ 
$1410^{-308}$ (computational limit of detection), two-sided Poisson test). When calculated for each

142 gene, we also observed mtDNA-encoded genes to have enriched mutation rates compared to

143 nuclear-DNA-encoded MSK-IMPACT genes $\left(P=2 \times 10^{-22}\right.$, two-sided Wilcoxon rank sum test):

144 only 2 MSK-IMPACT genes (TP53, KRAS) exhibited rates higher than that of the most mutated

145 mtDNA-encoded genes (Fig. 1f). Furthermore, the 13 protein-coding mtDNA genes exhibited a

146 4.2-fold higher rate of truncating variants which disrupt the reading frame (i.e. nonsense

147 mutations and frameshift indels) compared to truncating mutations among 185 known tumor

148 suppressor genes in the MSK-IMPACT cohort (which also included splice-site variants which

149 cannot arise in the mitochondrial genome due to the lack of introns) $\left(P=9 \times 10^{-5}\right.$, two-sided

150 Wilcoxon rank sum test) (Fig. 1g), and a 6.7-fold higher rate of non-truncating, non-synonymous

151 mutations (collectively referred to here as "missense" mutations) than 168 MSK-IMPACT

152 oncogenes $\left(P=6 \times 10^{-9}\right)$ (Fig. 1 h). In total, $11.9 \%$ of tumors across all cancers (95\% $\mathrm{Cl}: 11.0-$

$15312.9 \%$ ) harbored a truncating mtDNA variant absent in the patient's matched normal sample. In

154 contrast, only $0.15 \%$ of normal blood samples exhibited a truncating variant $(95 \% \mathrm{Cl}$ : $0.13-$

$1550.17 \%$ ) based on a recent analysis of $\sim 200,000$ mtDNA genomes ${ }^{14}$ (Fig. 1i). The rate of

156 truncating mutations in mtDNA genes in tumors therefore represents an 80-fold increase

157 compared to truncating mutations observed in normal human genomes (SI table 2.) Of the 619

158 truncating mutations we observed, 196 (32\%, 95\% binomial $\mathrm{Cl}: 28-35 \%)$ had $>80 \%$

159 heteroplasmy despite underlying infiltration of the bulk tumor by normal stromal and immune

160 cells, indicating that a significant number of tumors are dominated by a highly dysfunctional

161 mitochondrial genotype. Furthermore, high heteroplasmy truncating variants were significantly

162 more common than high-heteroplasmy silent mutations (139/555, 25\%, 95\% Cl: 21-29\%)

$163(P=0.01$, two-sided Fisher's exact test) under predominantly neutral selection.

\section{Truncating Mutations Preferentially Target Complex I at Homopolymeric Hotspots}

166 The physiologic response to genetic and pharmacologic inhibition of mitochondrial respiration

167 depends strongly on which mtDNA-encoded complex $(\mathrm{Cl}, \mathrm{CIII}, \mathrm{CIV}, \mathrm{CV})$ is disrupted, implicating

168 OXPHOS complex as a potential determinant of selective pressure for mutation. We therefore

169 investigated the somatic mutation rate according to the OXPHOS complex, controlling for the

170 relative length of mtDNA coding for genes in each complex and uneven coverage within each

171 sequenced sample. This revealed a striking dichotomy in the relative enrichment of mutations in

172 each complex. Truncating variants (nonsense mutations and frame-disrupting indels) arose at a

1732 -fold or greater rate in complex I relative to the other complexes $(P=0.001$ for least significant

174 comparison, two-sided Poisson test) (Fig. 2a). No difference in mutation rate between 
complexes was observed for silent mutations, consistent with a lack of differential selective pressure for synonymous protein changes ( $P=0.5$ for most-significant comparison). Unlike variants in other complexes, truncating variants in $\mathrm{Cl}$ demonstrated higher heteroplasmy (variant allele frequency) than silent variants $\left(P=1 \times 10^{-6}, \mathrm{Cl}\right.$; most significant for other complexes, $P=0.4$, two-sided Wilcoxon rank sum test) (Fig. 2c). Finally, complex $\vee$ genes (MT-ATP6 and MT-ATP8) demonstrated significantly lower rates of truncating but not synonymous mutations. The findings above were recapitulated in an independent cohort, composed of a distinct mixture of cancer types, of $\mathrm{N}=1,951$ whole-genome sequenced tumors from the PCAWG dataset, after excluding samples overlapping with our own cohort (Fig. 2b). Tumors of different lineages exhibited wide variability in the incidence of truncating mutations, with $\leq 5 \%$ of some cancer types affected by truncating mutations (sarcomas, gliomas), to $20 \%$ or greater of other cancer types (renal cell, colorectal, thyroid) (Fig. 2d). In renal, thyroid, and colorectal cancers, the high burden of truncating variants was driven by a specific enrichment for mutations to complex I (Qvalue $<0.01$, two-sided McNemar's test) (Fig. 2e). Truncating variants in these three cancers affected between $20-30 \%$ of all samples, corresponding to a prevalence on the same order or exceeding that of common tumor suppressors in these diseases. Taken together, these data indicate that the functional consequence of mtDNA variants and the complex they target are key determinants of the pattern of somatic mtDNA mutations. Additionally, they suggest that disruption of complex $\mathrm{V}$, which would fundamentally impair mitochondrial ATP production independent of the activity of all other OXPHOS complexes, is not tolerated.

Unexpectedly, we observed that truncating mutations frequently arose at the same genomic locus, analogous to well-described hotspot mutations that accumulate in nuclear cancer driver genes and often reflect selective pressure ${ }^{15,16}$. These apparently recurrent alleles were exclusively indels, rather than nonsense mutations, characterized by a homopolymeric sequence context. We therefore developed an approach to detecting recurrent mutations at homopolymeric loci by modeling incidence of frame-shift indels at each locus as a function of their base-pair length (see Methods). Six single-nucleotide repeat loci (out of seventy three loci of 5 or more base-pairs in length) in MT-ND1 (c.3,566-3,571, $n=32$ ), MT-ND4 (c.10,947$10,952, n=25$; c. 11,032-11,038, $n=34$; and c.11,867-11,872, $n=50$ ), and MT-ND5 (c.12,385$12,390, n=23$ and c.12,418-12,425, $n=73$ ) accumulated mutations at a rate above null expectation ( $Q$-value<0.01, Fig. 2f). Homopolymer hotspots only arose at single-nucleotide loci of at least $6 \mathrm{nt}$ in length ( $P=0.0002$, two-sided Fisher's exact test), were composed of $A$ or $C$ homopolymer repeats, and exclusively encoded subunits of complex I. Importantly, other 
homopolymers of equivalent length $(\geq 6)$ and nucleotide content exist both in complex I and complex III/IV/N but did not exhibit recurrent mutations, indicating a high degree of specificity to hotspot positions (Fig. 2g). These six homopolymeric repeat loci collectively accounted for $40 \%$

212 of all truncating variants observed in our data (95\% binomial Cl: $36-44 \%)$, and 57\% (95\% Cl:

$21352-62 \%$ ) of frame-shift indels overall. Notably, recurrent loss-of-function frameshift indels have

214 been observed at these sites as early driver mutations in rare, often benign renal oncocytomas

215 17; however we observed mutations at these loci to be a pervasive phenomenon across tumor

216 lineages (Supplementary Fig. 2a). Homopolymeric hotspot mutations arose in the PCAWG

217 cohort (after excluding any samples overlapping with our cohort) at a rate highly consistent with

218 the TCGA cohort (Pearson's $r=0.95$ ), indicating that the indels detected in TCGA at hotspot

219 loci were not artifacts due to calling variants in microsatellite regions with poor coverage

220 (Supplementary Fig. 2b). Moreover, the three most prevalently mutated of these homopolymer 221 loci in our dataset (c.11,032-11,038, c.11,867-11,872, c.12,418-12,425) intersected with 100-bp-

222 long windows enriched for frameshift indels identified in an analysis of 616 pediatric and 2,202

223 adult tumors (527 of which were from TCGA), highlighting the power of our approach to resolve

224 focal, recurrent alterations ${ }^{18}$. Although mutations at homopolymeric tracts have not been widely

225 described in the germline literature, the most recurrent hotspot (MT-ND5 c.12,418-12,425) has

226 been previously reported as the site of a germline frame-shift deletion (A12425del) in a pediatric

227 patient, where the de novo heteroplasmic deletion resulted in mitochondrial myopathy and renal

228 failure ${ }^{19}$.

Non-synonymous mutations and RNA variants arise as rare recurrent alleles with elevated

231 pathogenicity

232 The bulk of somatic variants we observed in mtDNA were non-truncating, non-synonymous

233 mutations, including missense mutations, in-frame indels, translation start site mutations and

234 non-stop mutations (collectively variants of unknown significance or VUS, $73.2 \%$ of $n=4,381$

235 variants, Fig. 3a). Interestingly, non-synonymous variants were again depleted in CV relative to

236 other complexes, suggesting that $\mathrm{CV}$ is intolerant both to truncating variants and to presumably

237 less-disruptive non-synonymous mutations. Using the APOGEE framework to evaluate the

238 functional consequence of mutations to protein-coding mtDNA genes ${ }^{20}$, we found that somatic

239 VUSs were twice as likely to be predicted pathogenic compared to germline polymorphisms

240 observed among $200 \mathrm{~K}$ normal samples from the HelixMTdb dataset $(39.5 \%$ of somatic-only

241 variants compared to $20.4 \%$ of germline-arising, $P=6 \times 10^{-14}$, two-sided Wilcoxon rank sum test,

242 Fig. 3b). Furthermore, when considering all possible mtDNA variants excluding germline 
243 polymorphisms (i.e. the complete set of all possible somatic variants), VUSs observed in tumors

244 were more pathogenic than the set of possible somatic variants which never arose in tumors,

245 suggesting that somatic VUSs are more pathogenic than expected by random chance. We next

246 evaluated the tendency for VUSs to target specific complexes of the ETC (this necessarily

247 reduced the types of VUSs to protein-coding variants, including missense, in-frame indels, and

248 a small number of translation start site and nonstop mutations). In contrast to truncating

249 variants, protein-coding VUSs were most frequent in CIII $\left(P=1 \times 10^{-7}\right.$ for least significant

250 comparison, two-sided Poisson test, Fig. 3c), whose functional integrity as a site for ubiquinol

251 oxidation has recently been described as essential for tumor cell proliferation ${ }^{21}$, although as with

252 truncating variants VUSs to CV subunits were still depleted compared to the other complexes

253 ( $P=0.01$ for least significant comparison). These observations were validated using data from

254 PCAWG (Fig. 3d). Together, these findings suggest that tumors preferentially accumulate

255 somatic missense mtDNA mutations in a manner dictated by OXPHOS complex, possibly driven

256 by their capacity to disrupt mitochondrial function due to their elevated pathogenicity.

257 Furthermore, they support the hypothesis that a purifying selection exists against variants (both

258 truncating and VUSs) that compromise physiological function of complex VIATP Synthase.

Single nucleotide variants (SNVs) were far less recurrent than homopolymer indels $(P=0.01$, two-sided Wilcoxon rank sum test among distinct variants mutated in $>=3$ tumors, Fig. $3 e$ ). However, we nevertheless observed a small number of loci with recurrent non-truncating variants. recurrent mutant loci. We developed a statistical test for recurrence of these loci, and identified 7 SNV hotspots in the mitochondrial genome ( $Q<0.01$, Fig. 3f), including 3 in proteincoding genes (all in complex I), 3 in ribosomal RNAs (all in MT-RNR2), and 1 in a tRNA (MT$T L 1)$ (see Methods). In contrast to the high fraction of truncating mutations which are explained by a relatively small number of hotspot alleles, hotspot SNV mutations collectively accounted for $1.6 \%$ of all VUSs; the vast majority of VUSs were non-recurrent, usually arising in a single sample. Furthermore, 0/33 mutations arising at the three protein-coding hotspot positions were nonsense mutations introducing an early stop codon, suggesting either the mutagenic

271 mechanism generating homopolymeric indel hotspots has a high degree of specificity, or that

272 truncating hotspots themselves may engender unique phenotypes beyond conventional loss-of-

273 function.

275 Mitochondrial tRNAs (mt-tRNAs) are commonly mutated in the context of germline mitochondrial 276 disease. Interestingly, the somatic hotspot MT-TL $1^{A 3243 G}$ (somatically mutated in 6 patients) is 
also the causative variant of around $80 \%$ of MELAS disease cases and approximately $30 \%$ of all mtDNA disease ${ }^{22,23}$. We additionally observed mutations clustered in adjacent positions $3242(n=5)$ and $3244(n=4$, recently described as a recurrent mutation in Hürthle cell carcinoma of the thyroid ${ }^{24}$ ), suggesting that recurrent mutations in MT-TL1 could affect a common secondary structure element. Mitochondrial tRNAs adopt a relatively conserved cloverleaf structure upon folding, and mutations to mt-tRNAs are known to disrupt the function of specific secondary structure elements. We therefore sought to test whether any positions of the tRNA cloverleaf structure were enriched for somatic mutations in tumors. We aligned all tRNA mutations according to their position in the canonical mitochondrial tRNA structure and developed a statistical approach to identify enrichment in specific secondary structure elements (see Methods). This analysis identified position 31 in the anti-codon stem of the folded tRNA molecule as a site of recurrent mutation across mt-tRNAs $\left(Q=4.7 \times 10^{-4}\right.$, Fig. $\left.\mathbf{3 g}\right)$, which we further validated using the non-TCGA subset of PCAWG samples $(Q=0.014$, Supplementary

Fig. 3a). Interestingly, position 31 was observed to be mutated at an 8-fold higher rate in tRNAs encoded on the light-strand (e.g. MT-TC, $n=5$; MT-TP, $n=4$; MT-TA, $n=3$ ) compared to heavystrand-encoded tRNAs ( $P=2 \times 10^{-4}$, two-sided Fisher's exact test). As a group, mutations at structural position 31 were predicted to be more pathogenic by MITOTIP relative to mutations at other tRNA positions (Fig. 3h), and in the case of $M T-T A^{T 5628 C}(n=3)$ are associated with the mitochondrial disease chronic progressive external ophthalmoplegia (CPEO) ${ }^{25}$. In analogy to the recurrent mutation of conserved amino acid residues in domains of homologous proteins ${ }^{26}$ or within 3-dimensional regions of folded protein structures ${ }^{27}$, these data suggest that specific structural features of mt-tRNAs may undergo recurrent mutation and impair normal mitochondrial physiology.

To understand the potential function of rare protein-coding SNV hotspots in mtDNA, we focused on a recurrent mutation at $M T-N D 1^{R 25}$, which was identified somatically in 11/10,132 TCGA patients $(0.11 \%)$, and $5 / 2,836$ PCAWG patients $(0.18 \%)$. All 16 instances resulted in a substitution of arginine $(R)$ with glutamine $(Q)$, encoded by a $G>A$ substitution at position 3380 . $M T-N D 1^{R 25 Q}$ was previously described in a case report as the causative variant in the development of MELAS in a mitochondrial disease patient ${ }^{28}$, but was never observed among 200K normal samples, where the mutant alleles at residue R25 always produced synonymous mutations (A3381G, $n=57$ ). Residue R25 is conserved across vertebrates ${ }^{28}$, and is part of a cluster of charged residues in complex I which form a structural bottleneck in the ubiquinone binding tunnel leading to the $Q$ binding site ${ }^{29}$. This led us to hypothesize that the R25Q mutation 
311 could potentially disrupt the site, impacting ubiquinone : complex I binding kinetics and/or Q-site

312 substrate specificity, impeding the downstream electron transport chain. We therefore modelled

313 the effect of $M T-N D 1^{\mathrm{R} 25 \mathrm{Q}}$ using a recent, high resolution structure of the mammalian. This

314 analysis highlighted changes to the local charge environment due to loss of the relatively bulky,

315 positively charged arginine sidechain. Due to the location of this substitution within the $Q$

316 binding tunnel, this is predicted to significantly impact function (Fig. 3i). Focusing on colorectal

317 tumors, which demonstrated the largest numbers of tumors harboring $M T-N D 1^{R 25 Q}$ ( $n=8$ tumors

318 total), we examined whether the presence of $M T-N D 1^{R 25 Q}$ was associated with a particular

319 transcriptional signature. Relative to mtDNA-wild-type tumors, we observed that MT-ND1 ${ }^{R 25 Q}$

320 tumors were characterized by upregulation of MYC targets and oxidative phosphorylation, and

321 downregulation of gene signatures associated with hypoxia, IL2/STAT5 signaling, TNFa

322 Signaling via NFKB (Fig. 3j). These data suggest that MT-ND1 ${ }^{R 25 Q}$ promotes a transcriptional

323 phenotype characterized by increased mitochondrial metabolism and suppressed expression of

324 innate immune genes.

Mitochondrial genotype underlies a lineage-agnostic transcriptional program

327 Given the lineage specificity underlying both truncating variants and truncating/SNV hotspots, we studied the overall burden of distinct classes of mtDNA variants (i.e. producing a truncating, missense, synonymous, tRNA or rRNA variant) across cancer types. Restricting our analysis to well-covered samples including coverage over all homopolymeric hotspots (see Methods), we found that the fraction of mutant samples across cancer types ranged from approximately $23 \%$ of leukemias (95\% binomial $\mathrm{Cl}$ : $13-35 \%)$ to as high as $80 \%$ of thyroid cancers (95\% $\mathrm{Cl}: 63-92 \%$ )

(Fig. 4a). Moreover, we observed no correlation between the fraction of well-covered samples in a cancer type and the proportion of samples with a somatic mtDNA mutation (Supplementary Fig. 4a), indicating that the highly variable incidence of different somatic variants across cancer types was not biased by their differing sequencing coverages. This extensive variation suggests tumor lineages may be subject to different degrees of selection for or against mtDNA mutations, consistent with the extensive variability of $\mathrm{dN} / \mathrm{dS}$ ratios previously described in somatic mtDNA mutations derived from whole genome sequencing of the TCGA ${ }^{5}$.

Truncating mtDNA mutations approaching homoplasmy (>90\% heteroplasmy) were identified in

342 nearly all cancer types, despite the tendency for stromal and immune cell infiltration to suppress 343 apparent tumor cell heteroplasmy, suggesting that even cancers in which mtDNA mutations are 344 uncommon may still contain rare instances of individual tumors with highly mutant mitochondria. 
In renal and thyroid tumors, truncating mtDNA mutations have historically been associated with the development of oncocytic neoplasia, whereby tumor cells accumulate dysfunctional mitochondria ${ }^{30,31}$. That truncating mutations induce a morphologically similar response in two different tissue lineages suggests that cells may adopt a lineage-agnostic adaptation to the presence of a truncating mutation. To evaluate if truncating mutations induced functionally similar consequences across different tumor lineages, we compared the gene expression profiles of tumor samples with truncating mtDNA variants to tumor samples with wild-type mtDNA (harboring no nonsynonymous somatic mutations in protein-coding or RNA genes, see Methods, Fig. 2f). In half of all cancer types, tumors harboring truncating mutations exhibited a conserved expression program characterized by upregulation of genes associated with oxidative phosphorylation and downregulation of genes associated with TNFa via NFKB signaling (Fig. 4b and Supplementary Fig. 4b). Critically, these expression programs were evident in cancer types such as glioma and mesothelioma, where the proportion of samples with a truncating variant was comparatively low (Fig. 4c). These data suggest that, even in cancer types where mtDNA mutations are rare, truncating mtDNA mutations produce similar phenotypic outcomes.

Given that the hotspot $M T-N D 1^{R 25 Q}$ exhibited an expression program resembling truncating variants, we investigated the generic transcriptional consequences of mtDNA VUSs (see Methods). Compared to truncating variants, fewer genesets demonstrated lineage-agnostic changes in samples with VUSs. As with truncating variants, the most upregulated geneset in VUS-harboring tumors was Oxidative Phosphorylation (increased in 5/18 cancer types) (Supplementary Fig. 4c), but the magnitude of this enrichment was attenuated relative to truncating variants. Notably, several cancer types, such as colorectal cancer, demonstrated a lineage-specific pattern of gene expression changes, suggesting that mtDNA VUSs are capable of eliciting a phenotype in specific cancer types.

To examine the translational value of mtDNA genotype, we determined the association between

373 mtDNA mutation status and clinical outcome (overall survival) across cancer types. Using

374 univariate Cox proportional-hazards regression, for each cancer type we determined the effect

375 size and significance of both mtDNA truncating variants and VUSs compared to samples with 376 no somatic mtDNA variants (wild-type). Colorectal cancer demonstrated the largest (by effect377 size) significant association between overall survival time and mtDNA genotype (colorectal 378 patients with VUSs had a hazard ratio of $0.47(95 \% \mathrm{Cl} 0.03-0.75)$ compared to those with wild- 
379 type mtDNA, Q-value=0.02, Cox proportional-hazards regression) (Fig. 4d). Notably, VUSs in

380 colorectal cancer also associated with a unique transcriptional down-regulation of multiple

381 genesets including TNFa via NFkB, Hypoxia and Complement (Supplementary Fig. 4c, Fig.

$3823 \mathrm{j}$ ), further suggesting a cryptic phenotype of these variants in affected tumors. We additionally

383 observed a weak association between mitochondrial genotype and underlying molecular

384 subtype ${ }^{32}$, with some enrichment of mtDNA mutations in the canonical subtype CMS2 of

385 colorectal tumors(Supplementary Figure 4d). We therefore further evaluated if mtDNA

386 mutations may be prognostically meaningful in colorectal cancer, using a multivariate analysis to

387 control for known prognostic clinical and genomic covariates. Among 344 stage 1-3 colorectal

388 cancer patients, the presence of mtDNA alterations was significantly associated with better

389 overall survival compared to wild-type samples ( $P=0.002$, Kaplan-Meier test), with patients

390 whose tumors harbored VUSs having the best prognosis, and those with truncating variants

391 having an intermediate improvement (Fig. 4e). This association remained significant after

392 controlling for clinically-relevant prognostic covariates (i.e. age, cancer stage, primary site, MSI-

393 status, consensus molecular subtype and the presence of established nuclear-encoded

394 genomic driver mutations) ${ }^{32,33}$ in a multivariate analysis. VUSs again had a significantly

395 protective association compared to wild-type (Hazard ratio=0.18, 95\%; Cl: 0.08-0.44; Q-

396 value $=0.001$, Cox proportional-hazards model); truncating variants had an intermediate effect

397 (HR=0.38, 95\% Cl: 0.15-0.97; Q=0.18) (Fig. 4f). These data together suggest that somatic

398 mtDNA mutations are associated with a clinically and molecularly-distinct class of colorectal

399 tumors, and that the functional consequence of an mtDNA mutation is a determinant of its

400 clinical significance.

\section{Discussion}

404 Although recent evolutionary data suggests that mtDNA mutations may be under positive

405 selection in cancers of the kidney and thyroid ${ }^{5}$, the broader significance of somatic mtDNA

406 mutations in cancer remains a point of confusion and debate. Drawing inspiration from analyses

407 describing hotspots of somatic mutations in the nuclear DNA of tumors, we studied the

408 recurrence of mutant mtDNA alleles. The discovery that OXPHOS complex shapes mtDNA

409 mutation patterns in a manner that produces mutation hotspots, in connection with orthogonal

410 data on the structural consequences, transcriptomic effects and clinical significance of these

411 alleles in patients with germline mtDNA disease, supports the hypothesis that mitochondrial

412 respiration is perturbed across many tumors. 
414 Our results indicate that OXPHOS complex, tissue lineage, and mutation consequence

415 collectively shape the incidence and putative function of mtDNA mutations. Whereas previous

416 studies have demonstrated localized regions of mtDNA with elevated somatic mutation rate in

417 tumors, these works have generally been underpowered to probe phenotypic differences

418 between alleles. Our data reveal that truncating mutations preferentially impact complex I, and

419 that non-synonymous mutations of all classes are depleted in complex $\mathrm{V}$. This suggests that

420 cancer cells can better tolerate, or perhaps even utilize, loss of complex I and the associated

421 metabolic consequences (e.g. NAD+:NADH changes), whereas loss of capacity for ATP

422 synthesis through complex $\mathrm{V}$ mutations appears to be negatively selected against. That CIII

423 demonstrates elevated rates (relative to other complexes) of missense mutations, but not

424 truncating mutations, is consistent with its essential role in ubiquinol oxidation and suggests that

425 weak disruption of $\mathrm{CIII}$ is preferential for clonal expansion in tumor cells ${ }^{21}$. Whether truncating

426 mutations in CIII and CIV promote different phenotypes in cancer cells relative to complex I loss

427 warrants further investigation.

429 There is substantial evidence that in particular subtypes of thyroid and kidney cancer, mtDNA

430 mutations are the root cause of metabolic adaptations and morphological (oncocytic) changes

431 associated with suppression of mitochondrial respiration ${ }^{34}$. What remains unclear is how to

432 extrapolate the function of truncating mutations in otherwise essential mtDNA genes to cancer

433 types where oncocytic tumors are rarely if ever observed but the fraction of samples harboring

434 these mutations is nevertheless substantial (e.g. colorectal cancers). Critically, our

435 transcriptional data suggests that, even in cancer types where truncating mtDNA mutations are

436 rare, they nevertheless promote a transcriptional program characterized by increased

437 expression of OXPHOS genes and downregulation of innate immune pathways. Because

438 homoplasmic loss of any gene in the mtDNA necessarily cripples the cell's ability to respire and

439 disrupts dependent metabolic pathways, these findings suggest that pathogenic and high

440 heteroplasmy mtDNA mutations potentially render a large fraction of tumors vulnerable to a

441 metabolic therapeutic intervention.

\section{Methods}

\section{Tumor and normal sample sequencing cohorts}


446 Tumor and matched normal sequencing data for TCGA samples were obtained from the GDC

447 Data Portal (https://portal.gdc.cancer.gov/). Briefly, all tumor and matched-normal barcodes

448 included in the MC3 MAF ${ }^{35}$ (https://gdc.cancer.gov/about-data/publications/pancanatlas) file

449 were converted to UUIDs using the TCGAutils R package (v1.9.3), and these UUIDs were

450 queried for whole-exome sequencing BAM files sliced for chrM using the GDC API. We then

451 queried the GDC Data Portal for RNA-Sequencing BAM files for TCGA tumors already with

452 whole-exome sequencing data. This process yielded paired tumor and matched-normal whole-

453 exome sequencing BAMs for 10,132 TCGA patients, of which 9,455 had additional RNA-

454 sequencing data. In addition to the raw sequencing data for TCGA samples from which we

455 called mtDNA mutations (see: Calling mitochondrial variants), we additionally obtained somatic

456 mitochondrial mutation calls for 2,836 whole-exome sequenced tumors from ICGC/PCAWG ${ }^{3}$, of

457 which 885 also had TCGA sequencing data. Nuclear somatic mutations for TCGA samples were

458 obtained from the MC3 MAF, subset for the samples for which mtDNA whole-exome

459 sequencing BAMs were available. Finally, mtDNA mutation calls for 195,983 normal samples

460 were obtained from the HelixMTdb cohort of sequenced saliva samples from healthy individuals 46114

\section{Annotating mtDNA regions included in our analysis}

464 Each mitochondrially-encoded gene's name, start/end positions and DNA strand was obtained 465 from Biomart for human reference genome GRCh38 (release 95). Subsequently, each mtDNA 466 position (1-16569) was annotated with its associated genetic information. Any mtDNA positions 467 located at the overlap of two genes were annotated only as associated with whichever gene 468 started first in numerical genomic position. Variants in non-genic mtDNA regions were excluded 469 in our analyses. To this end, we excluded any variants in the mtDNA Control Region (positions $4701-576,16,024-16,569)$ as well as 89 other non-genic positions. We similarly excluded variants in 471 hypermutated regions of mtDNA, including 302-316, 514-524, and 3,106-3,109). Following 472 these measures, the genomic length of mtDNA retained in our analyses was 15,354bp. (The

473 complete list of 16,569 mtDNA positions and their annotated reasons for exclusion is provided in

474 SI table 1.)

475

\section{Calling mitochondrial variants}

477 Mutations to the mitochondrial genome were obtained from variants called by both of two 478 independent variant-calling pipelines. In the first pipeline, Mutect2 (GATK v4.1.2.0) ${ }^{36}$ was used

479 to call variants in chrM in tumor and normal samples individually, the results of which were 
subsequently intersected to obtain variants called supported in a given patient's tumor and matched normal samples. Briefly, Mutect2 was run in mitochondrial-mode for each patient's tumor and normal sample independently against human reference genome GRCh38 (with

483 minimum base quality-score 20 , minimum mapping quality 10 , aggressive pcr-indel model, and

484 other standard quality control arguments for paired-end reads). Artifacts were subsequently

485 removed using GATK FilterMutectCalls (GATK v4.1.2.0) ${ }^{36}$, and multi-allelic sites were split into 486 individual variants using the norm function from bcftools (v1.9) ${ }^{37}$. The resulting tumor and 487 normal VCF files were then merged using gatk HaplotypeCaller (GATK v4.1.2.0) ${ }^{36}$, to annotate 488 variants in the tumor VCF with their coverage in the normal sample. The resulting VCF was converted to a MAF file using vcf2maf (v1.6.17, https://github.com/mskcc/vcf2maf). Finally, variants from the generated MAF file were then filtered out unless the variant allele was supported at least one read in both forward and reverse directions. In the second pipeline, samtools mpileup (v1.9) ${ }^{11}$ was used to generate a pileup file using variant-supporting reads with minimum mapping quality 20 and base alignment quality 10 . Reads failing quality checks or marked as PCR duplicates were removed. Variants were required to contain at least 2 variantsupporting reads in the forward and reverse direction. In each pipeline, variants were additionally filtered to ensure $\geq 5 \%$ variant allele frequency in the tumor, and tumor coverage $\geq 5$ reads. Variants identified by both pipelines were retained for further analysis. In rare cases, multiple indels were called in a sample within a homopolymeric region (single-nucleotide repeats of 5 or more basepairs), with distinct alt-read counts and VAF values, and identical read-depth values. These multiple indels were collapsed to a single representative indel call. Briefly, using the Mutect2 variant calls, whichever indel had the highest VAF in the tumor sample was taken as the representative indel. The count of alt-reads in both tumor and normal were replaced with their corresponding summed counts across the original multiple indels, and the VAFs in both tumor and normal were re-calculated from the new summed alt-read counts divided by the original read-depth. Finally, mutations were classified as of somatic origin according to the following criteria: Non-truncating variants (that is, all variant classifications

507 other than nonsense mutations and frame-shift indels) were classified as somatic if the matched

508 normal sample had a minimum coverage of 5 reads and 0 normal reads called the alternate

509 allele. Truncating variants in the tumor sample were assumed to be of somatic origin. All other 510 variants were not classified as somatic and excluded from this study.

\section{Nuclear mutational data and annotation}


513 Somatic mutations in nuclear-encoded cancer-associated genes for TCGA samples were

514 obtained from the PanCanAtlas MC3 MAF file. Mutations in this file were subset for those

515 among the 468 genes on the MSK-IMPACT clinical sequencing panel ${ }^{13}$. The MAF file was

516 annotated for known, likely, and predicted oncogenic driver mutations using the MAF-Annotator

517 tool provided by OncoKB ${ }^{38}$ (https://github.com/oncokb/oncokb-annotator). Mutations annotated

518 by OncoKB as "Oncogenic", "Likely Oncogenic" or "Predicted Oncogenic", previously

519 determined cancer hotspot mutations ${ }^{15,16}$, or truncating variants to tumor suppressor genes (i.e.

520 frame-shift indels, splice-site and nonsense mutations) were classified as potential driver

521 alterations.

\section{Calculating tumor mutational burden in mtDNA or nuclear DNA}

524 Tumor mutational burden (TMB) was calculated for cohorts of tumors subset for various

525 genomic regions, including: 1) individual mitochondrial- or nuclear-encoded genes; 2) mtDNA

526 genes grouped by OXPHOS complex I, III, IV, or V; 3) the entire mitochondrial genome

527 (excluding non-genic and polymorphic regions); 4) a set of known nuclear-encoded tumor

528 suppressor genes; and 5) a set of known nuclear-encoded oncogenes. In each case, TMB was

529 calculated as the total number of somatic mutations among the relevant collection of tumors

530 divided by the total genomic length sequenced in these tumors (in Mbps). For TMBs calculated

531 from mutations called in off-target sequencing data (i.e. mtDNA variants in TCGA samples), the

532 total genomic length sequenced was the number of the genomic positions with sufficient

533 coverage to call somatic variants (5+ read coverage in both tumor and normal sample),

534 summed across all samples. For TMBs calculated from targeted regions (nuclear DNA; mtDNA

535 in PCAWG samples), the total genomic length sequenced was the length of the targeted region

536 (entire gene for mtDNA, exonic regions for nuclear DNA) multiplied by the number of samples.

537 Error bars for TMBs were calculated as 95\% Poisson exact confidence intervals for rates, using

538 the total number of mutations as the count of events, and the genomic length sequenced in $\mathrm{Mb}$

539 as the time at risk.

\section{Identifying hotspot positions for mitochondrial variants}

542 We identified mtDNA positions with statistically recurrent single-nucleotide variants (SNVs) by

543 comparing the observed proportion of mutations at an individual position (out of the total number

544 of mutations acquired in its gene) to a rate of mutations at the position expected by chance with

545 a one-sided binomial test. The probability for SNVs at each position of a gene $P_{\text {pos,gene }}$ was

546 modeled as a bernoulli trial, where the likelihood of a mutation arising at a given position by its 
547 mutability relative to the mutability of all other bases in the gene: $P_{\text {pos, gene }}=\frac{\mu_{\text {pos }}}{\mu_{\text {gene }}}$. Consistent

548 with previous work ${ }^{15}$, we estimated the mutability for each position as a function of its

549 trinucleotide context. That is, for each position, it's mutability $\mu_{\text {pos }}$ was calculated as the count of

550 SNVs matching the trinucleotide context of the position of interest $s_{\text {pos }}$, out of the total count of

551 SNVs anywhere in the mitochondrial genome $s_{\text {total }}$ (after excluding the control region and other

552 blacklisted regions). Due to the highly strand-specific mutation signatures we observed for

553 SNVs in mtDNA (Supplementary Fig. 1c), we used the complete set of 64 unique

554 trinucleotides in order to retain this information when calculating the mutability for each position,

555 rather than collapsing the central nucleotide to $\mathrm{C}$ or T resulting in the conventional 32 unique

556 trinucleotides. As the proportion of patients for which a given position had sequencing coverage

557 in paired tumor and normal samples linearly affects the likelihood of observing a somatic

558 mutation at the position, the mutability of a position was adjusted to control for this by

559 multiplying it by the ratio of the number of samples with paired tumor-normal sequencing

560 coverage at the position $C_{\text {pos }}$ out of the total number of samples $N_{\text {samples }}$, so that $\mu_{\text {pos }}=$

$561 \frac{s_{\text {pos }}}{s_{\text {total }}} \times \frac{C_{\text {pos }}}{N_{\text {samples }}}$. The mutability associated with the gene was calculated as the sum of each

562 position's trinucleotide mutability. Therefore, for a gene $L$ basepairs in length: $\mu_{g e n e}=$

$563 \sum_{p o s=1}^{L} \mu_{p o s}$. The final parameter for the binomial test (i.e. the likelihood for a mutation in a gene

564 to arise at the given position by chance) was therefore $P_{p o s, g e n e}=\frac{\mu_{\text {pos }}}{\mu_{\text {gene }}}$. Each position mutated

565 in 5 or more samples in each gene was subsequently tested for statistically enriched mutations

566 by comparing its observed number of mutations out of the total number of mutations in the gene

567 to this binomial parameter using a right-tailed binomial test. The full list of generated $P$-values

568 across all genes were then corrected for multiple hypothesis testing.

\section{Homopolymer hotspots for indels}

571 To identify homopolymer regions with statistically enriched rates of insertions and deletions

572 (indels), we modeled the proportion of samples with indels across all homopolymers as a

573 function of the homopolymer region's width (i.e. the number of repeated nucleotides, from 5-8).

574 To this end, all single-nucleotide repeats of 5 or more basepairs were identified in the

575 mitochondrial reference genome, resulting in $\mathrm{N}=73$ unique homopolymer loci in whitelisted

576 coding mtDNA. We then modeled the fraction of frame-shift indels across 73 homopolymers

577 observed to arise at a specific homopolymer locus $h$ as a binomial process dictated by the

578 length of the homopolymer $I_{h}$ divided by the summed length of all homopolymers, such that the 
expected likelihood of a frame-shift indel arising at a homopolymer by chance is given by: $p_{h}=$

$\frac{l_{h}}{\sum_{i=1}^{73} l_{i} l_{i}}$. We then tested each homopolymer locus for enriched mutations with a one-sided

581 binomial test. That is, for each homopolymer locus, the number of bernoulli trials was the number of samples with complete sequencing coverage for the homopolymer region and two flanking basepairs; the number of successes was the number of samples with frame-shift indels at (or immediately adjacent to) the given homopolymer, and the fraction of successful trials was compared to the expected probability $p_{h}$.

\section{Hotspot positions in tRNA cloverleaf structure}

588 Positions of the tRNA cloverleaf secondary structure were individually tested for an enriched rate of SNVs at the equivalent aligned positions of the 22 mitochondrially-encoded tRNAs. A map of genomic positions in mitochondrial tRNAs to cloverleaf structure positions was provided by Mitotip 39 (https://github.com/sonneysa/MitoTIP/blob/master/Output/tRNA\%20data\%20and\%20scoring sc ored.xlsx) and used to assign SNVs at tRNAs to structural positions. Under the null hypothesis that mutations accumulate at structurally-aligned positions randomly, the proportion of SNVs aligning to a specific position in the tRNA cloverleaf should be approximately equal to the number of times the aligned position was sequenced at a sufficient depth in both tumor and matched normal samples to call somatic mutations, out of the total number of tRNA basepairs sequenced at sufficient depth across all samples at all structural positions. Therefore for a given position of the tRNA cloverleaf structure $p$, the number of SNVs observed across all tRNAs at

600 this aligned position $t_{p}$ out of $T$ SNVs across all positions of all tRNAs was tested for enrichment 601 using a one-sided binomial test, compared to an expected rate equal to the number of tRNA 602 bases aligned to this position sequenced at sufficient depth $b_{p}$ out of $B$ tRNA bases sequenced 603 at sufficient depth across all positions of all tRNAs.

\section{Classifying sample mtDNA variant status}

606 Each tumor sample was classified according to the presence and type of its somatic

607 mitochondrial variants. Because gaps in sequencing coverage may make existing variants 608 undetectable and result in the incorrect classification of such samples as "wild-type" for somatic 609 variants, we only attempted to classify samples with sequencing coverage in both tumor and 610 matched normal of at least $90 \%$ of the included region of mtDNA (referred to as "well-covered" 611 throughout). Furthermore, given the high incidence of truncating indels we observed at 6 
612 hotspot loci, we additionally required that these 6 loci were sequenced at sufficient coverage in

613 the tumor sample, to ensure that samples potentially harboring recurrent indels would be

614 excluded and not misclassified. Samples not meeting either of these conditions were classified

615 as having 'Unknown' mtDNA mutation status. The remaining samples were then classified

616 according to a decision tree as follows: Samples with any protein-truncating variants were

617 classified as 'Truncating'; remaining samples still unclassified with multiple mtDNA variants of

618 different types (among missense, rRNA, and tRNA variants) were classified as '2+ non-

619 truncating types'; remaining samples with tRNA mutations were classified as 'tRNA'; remaining

620 samples with rRNA mutations were classified as 'rRNA'; remaining samples with non-truncating,

621 non-synonymous protein-coding mutations as 'missense'; remaining samples with silent

622 mutations as 'Silent'; and finally samples still unclassified were classified as 'wild-type'. This

623 logic prioritizes minimizing annotation bias over conserving sample size, in order to meaningfully

624 compare the incidence of different variant types across samples. However, in our analysis of the

625 effect of mtDNA variants on differential gene expression or survival, we modified the logic to

626 prioritize conservation of sample size. To this end, in RNA-Seq and survival analyses, samples

627 with any observed truncating variants were classified as truncating, regardless of their

628 sequencing coverage.

Testing genesets for transcriptional dysregulation due to mtDNA variants

631 A matrix of estimated gene expression counts (RSEM values normalized to correct for batch

632 effects) for TCGA samples was downloaded from the TCGA PanCanAtlas ${ }^{35}$ supplemental data

633 (http://api.gdc.cancer.gov/data/3586c0da-64d0-4b74-a449-5ff4d9136611). Gene expression

634 estimates were rounded to integer values, and subsequently genes with zero estimated counts

635 in all samples were removed, as were genes with unknown gene symbols. To evaluate

636 differentially expressed genes between two groups of samples with different mtDNA variant type

637 (i.e. truncating vs wild-type samples colorectal samples), the rounded gene expression matrix

638 was subset for the relevant samples and input into the DESeq2 ${ }^{40}$ package in $\mathrm{R}$ using the

639 DESeqDataSetFromMatrix utility, along with a table of tumor sample barcodes with their

640 associated mtDNA classification. Differentially expressed genes were tested and their log-fold

641 change (LFC) values were shrunken using the apeglm ${ }^{41}$ package. $P$-values for all genes tested

642 were corrected for multiple-hypothesis testing with the Benjamini Hochberg method ${ }^{42}$. The

643 resulting data from this analysis were used to calculate a statistic for each gene equal to

$644 \log _{10}(Q$-value $) \times \operatorname{sign}\left(\right.$ LFC). All genesets from the mSigDB Hallmark geneset collection ${ }^{43}$ (v7.1) 
645 were then tested for significant up- or down-regulation based on this statistic for each gene

646 using the fgsea package ${ }^{44}$ in $\mathrm{R}$, with a minimum geneset size of 10 genes, a maximum size of

647500 genes, and 100,000 permutations.

Annotating genomic and clinical covariates in colorectal cancer survival analysis

650 Clinical data for TCGA colorectal cancer patients including: overall survival time/status, AJCC

651 pathologic tumor stage, age at diagnosis, sex, and tumor tissue site were obtained from the

652 TCGA FIrehose legacy data on cbioportal

653 (https://www.cbioportal.org/study/summary?id=coadread tcga). Clinical data was subset for

654 patients with sequencing data in the MC3 MAF. These data were then annotated with MSI

655 status (MSS, MSI-low, MSI-high) based on published data for patients where this was available

$656 \quad{ }^{45}$. AJCC Pathologic Tumor Staging data was collapsed into Stages I, II, III, IV, and Stage-IV

657 patients were excluded. The tumor site was encoded as "Right-colon" if the primary site was:

658 ascending colon, cecum, hepatic flexure, or transverse colon; or encoded as "Left-colon" for:

659 descending colon, sigmoid colon, or splenic flexure. Patients with tumor tissue from the rectum

660 were encoded as "Rectum" for their tumor site. The clinical data for each sample was then

661 annotated for the presence of known or likely nuclear-encoded driver alterations in

$662 \mathrm{KRAS/HRAS/NRAS,} \mathrm{BRAF,} \mathrm{APC,} \mathrm{SMAD4} \mathrm{and} \mathrm{TP53} \mathrm{as} \mathrm{based} \mathrm{on} \mathrm{mutation} \mathrm{calls} \mathrm{from} \mathrm{the} \mathrm{TCGA}$

$663 \mathrm{MC} \mathrm{MAF}^{46}$ (see: Methods "Nuclear mutational data and annotation"). Each patient in the

664 clinical data was then annotated as having a known/likely driver alteration in each of

665 KRAS/HRAS/NRAS (grouped into RAS), BRAF, APC, SMAD4 or TP53. The complete multi-

666 variate model use in the Cox proportional-hazards regression was therefore: Overall Survival

667 mtDNA-status + Age + Stage + Site + RAS + RAF + APC + SMAD4 + TP53 + Sex + MSI-status

$668+$ CMS-type.

669

670 Structural impact of $M T-N D 1^{R 25 Q}$ variant on complex I

671 The structural impact of the $M T-N D 1^{R 25 Q}$ variant was investigated using an electron-microscopy

672 derived structure of mitochondrial $\mathrm{Cl}$ in mus musculus (PDBID: 6G2J) ${ }^{29}$. The UCSF Chimera

673 software (v1.13.1) ${ }^{47}$ was used to insert the R25Q mutation using the swapaa command. The

674 ubiquinone binding tunnel was predicted using the CAVER Analyst (v2.0b) ${ }^{48}$ software run on

675 the wild-type PDB structure, starting from the side chain oxygen atom in Ndufs $2^{Y 108}$, and using a

676 minimum probe radius of $1.4 \AA$ as described by the authors ${ }^{49}$. Surface electrostatic charge for

677 wild-type and mutant structures were determined using the APBS software ${ }^{50}$

678 (http://server.poissonboltzmann.org/pdb2pqr) using default parameters, after subsetting the 
679 PDB structure for Mtnd1 (chain $\mathrm{H}$ ), and converting the resulting PDB file to PQR using

$680 \mathrm{PDB} 2 \mathrm{PQR}{ }^{51}$. All structure visualizations were generated using UCSF Chimera.

\section{Statistical analyses and figures}

683 All statistical analyses were performed using the $\mathrm{R}$ statistical programming environment (version

684 3.6.1). Protein structure figures were generated using UCSF Chimera, Kaplan-Meier plots and

685 Cox proportional hazard forest plots were generated with the survminer library in R, ETC

686 schematic (Fig. 1a) in Adobe Illustrator. All other figures were generated using the ggplot2

687 library in R. Unless otherwise noted, error bars for proportions are 95\% binomial Cls calculated

688 using the Pearson-Klopper method; error bars for rates (e.g. Mutations/Mb) are 95\% Poisson

689 Cls calculated with the pois.exact function from the epitools library in R. Unless otherwise noted,

$690 P$-values for difference in proportions were calculated using Fisher's exact tests or two-sample

691 Z-tests, and for difference in rates using Poisson exact tests. $P$-values were corrected for

692 multiple comparisons using the Benjamini-Hochberg method ${ }^{42}$ and reported as Q-values when

693 applicable.

694

Data and code availability

All relevant data and $\mathrm{R}$ code are available on GitHub with instructions to execute the code and regenerate all figures (https://github.com/reznik-lab/mtdna-mutations).

\section{Acknowledgements}

We thank the members of the Reznik and Taylor laboratories for discussion and support. We also thank Lydia Finley, Kivanc Birsoy, and Nicole Rusk for their feedback.

\section{Author contributions}

704 ANG, PAG, and ER conceived the study.MK, WKC, KL, AAH, and BST assisted with genomic

705 data analysis. ANG, PAG, and ER wrote the manuscript with input from all authors.

\section{Competing financial interests}

708 The authors declare no competing financial interests 

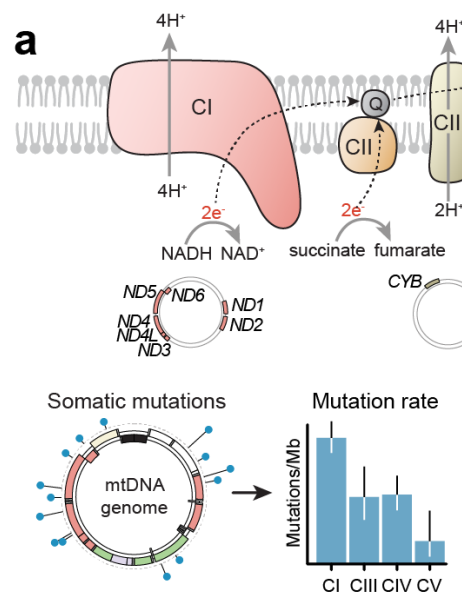

f

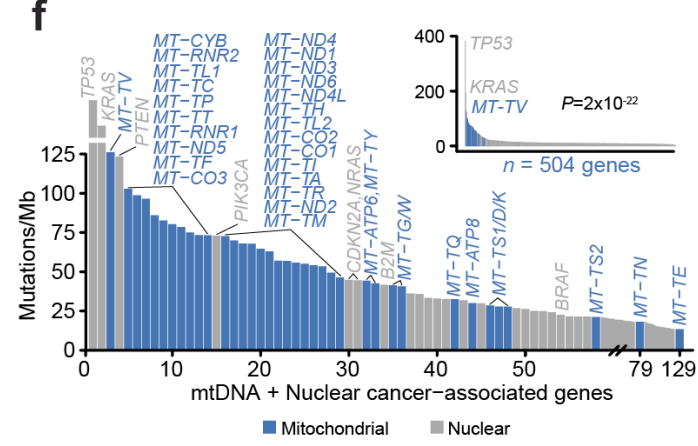

714

715

716

717

718

719

720

721

722

723

724

725

726

727

728

Fig. 1: mtDNA mutations are among the most frequent genomic alterations in cancer. a)
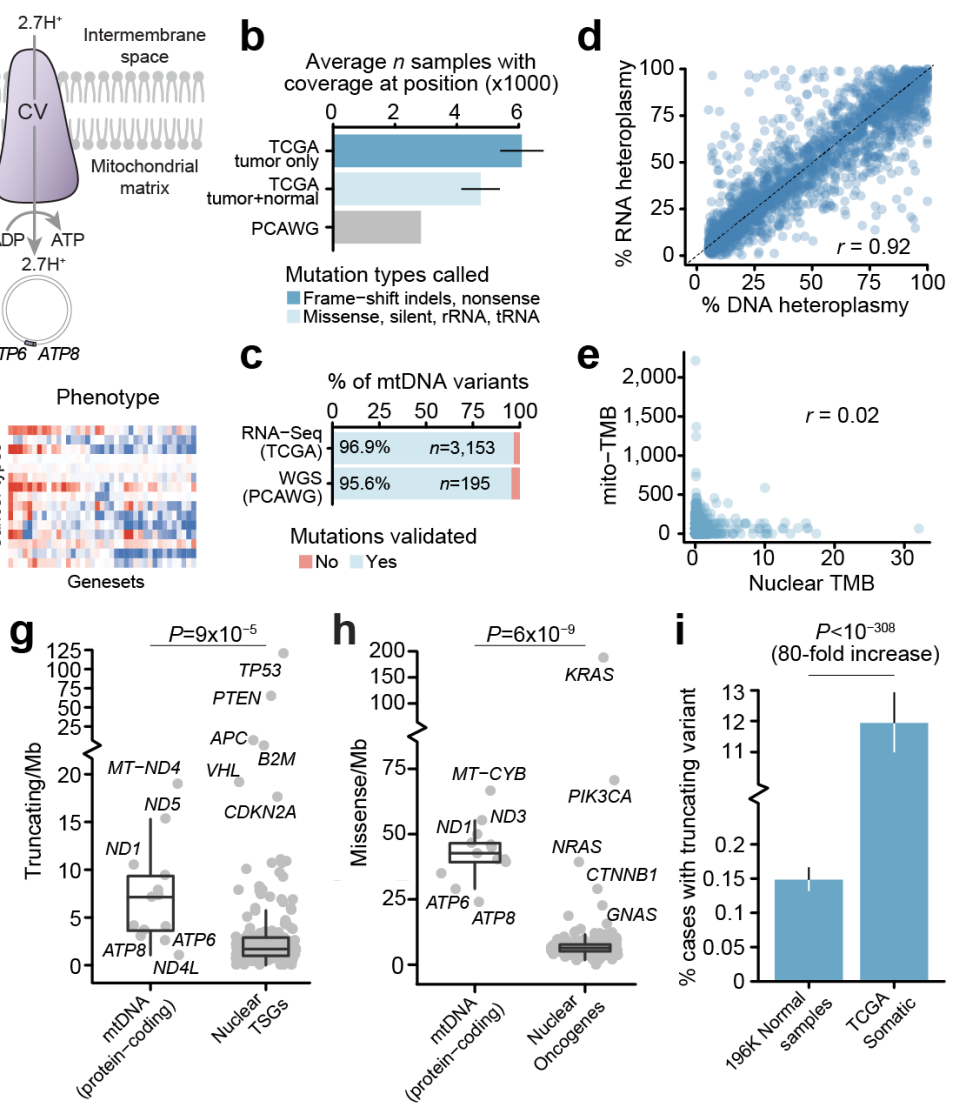

Schematic of oxidative phosphorylation (OXPHOS) system and project workflow. Top row, complexes I-V and their reactions. Center row: mtDNA genomic regions encoding protein subunits of the associated OXPHOS complex. Bottom row, overview of project workflow, in which somatic mutations in mtDNA genes are used to explore inter-complex differences, mutational recurrence and transcriptional phenotype associated with mitochondrial dysfunction.

b) Average number of tumors with sufficient coverage to call variants at a mtDNA position.

Truncating mutations were assumed to be somatic and therefore allowed for tumor-only variantcalling (dark blue), whereas non-truncating (protein-coding non-truncating, tRNA and rRNA mutations) required sufficient coverage in both tumor and matched normal samples (light blue). Gray, the number of whole-genome sequenced (WGS) samples from PCAWG for comparison. c) The percentages of variants called from off-target reads which were validated in either RNASeq or WGS data from the same tumors. d) The correlation between variant heteroplasmy as observed in RNA and DNA-sequencing ( $n=2,575$ mutations with coverage $\geq 30$ reads in both DNA and RNA). e) The correlation between tumor mutation burden (TMB, Mutations/Mb) among 
729 mtDNA (Y-axis) and nuclear-encoded cancer-associated genes (referred to simply as cancer

730 genes) (X-axis), $n=3,624$ well-covered pan-cancer tumors. f) Mutation rates (Mutations/Mb) of

731 individual mtDNA-encoded genes (blue) and nuclear-encoded cancer-associated genes (gray).

732 Inset plot: mutation rates among 504 genes with mtDNA genes highlighted. Outer plot: closeup

733 of the inset plot in the region containing all 37 mtDNA genes; commonly-mutated nuclear cancer

734 genes in this region are labeled for reference. $\mathbf{g}$ ) Comparison of truncating mutation rates

735 (truncating variants/Mb) between 13 mtDNA-encoded protein-coding genes and 185 nuclear-

736 encoded TSGs. h) Comparison of non-truncating mutation rate (nonsynonymous, non-

737 truncating variants/Mb) between 13 mtDNA protein-coding genes and 168 nuclear oncogenes. i)

738 Percentage of patients with truncating mtDNA variants either somatically (in TCGA tumor

739 samples) or germline (among 200K normal samples). 
a
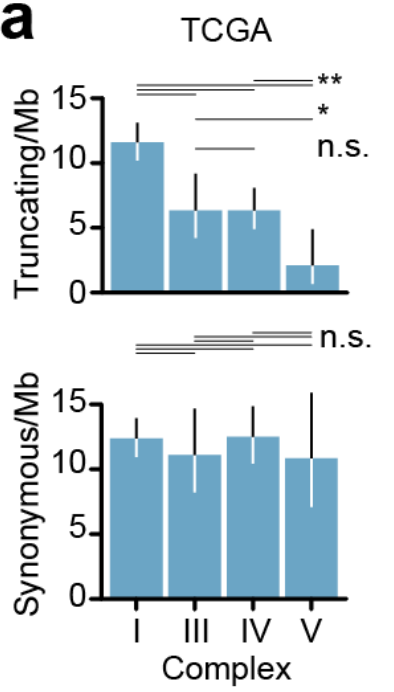

d

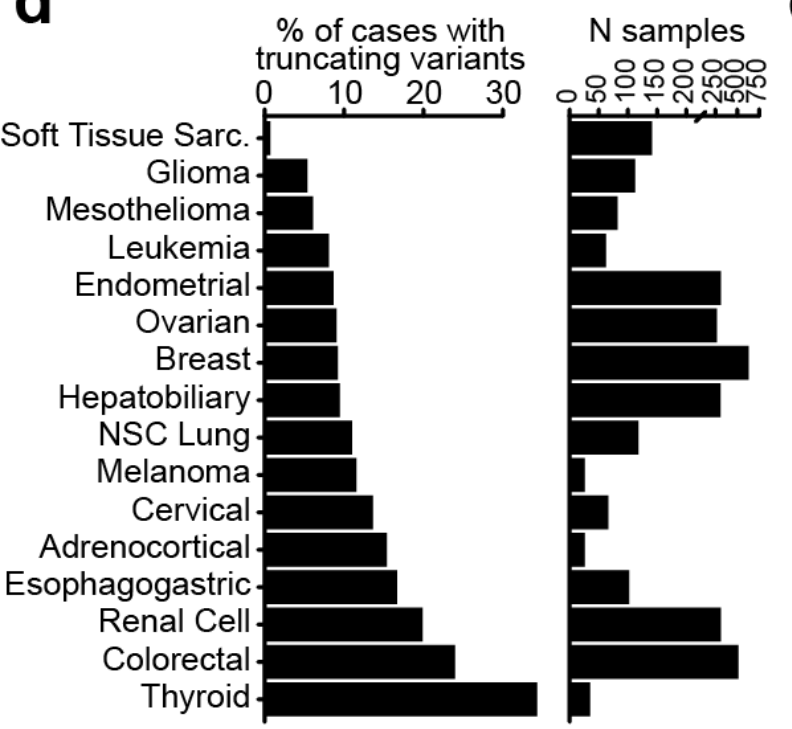

Complex with truncating variant
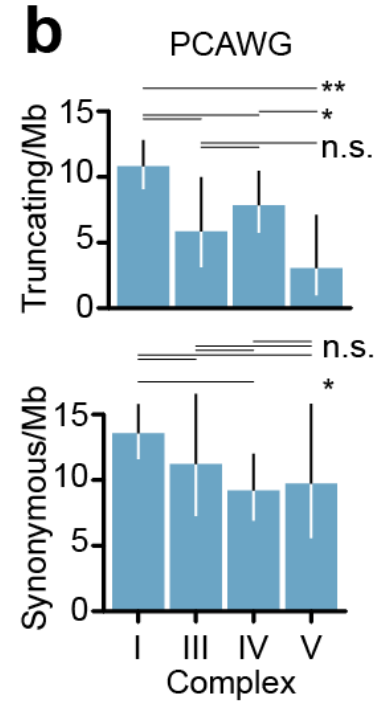

e

$\%$ of samples

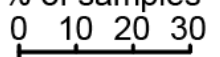

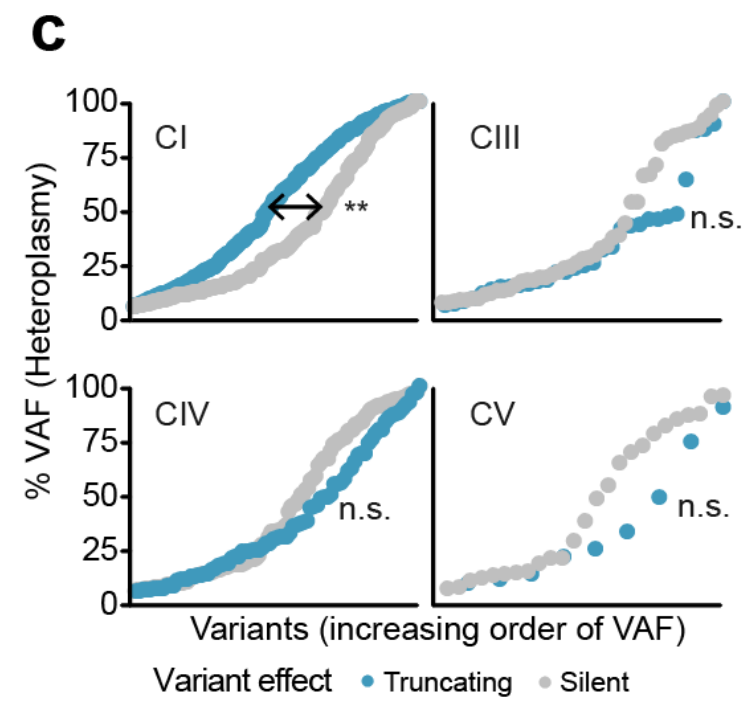

g
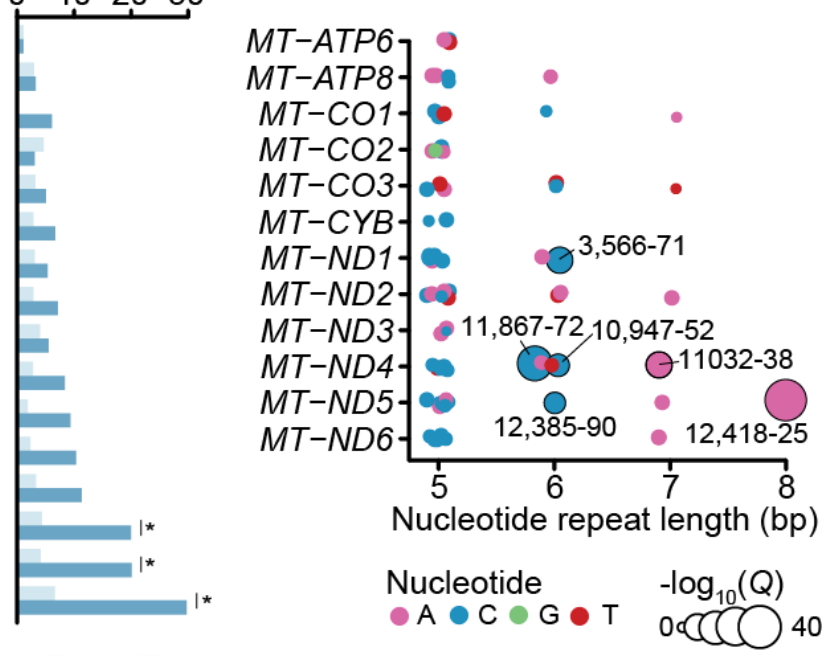

f

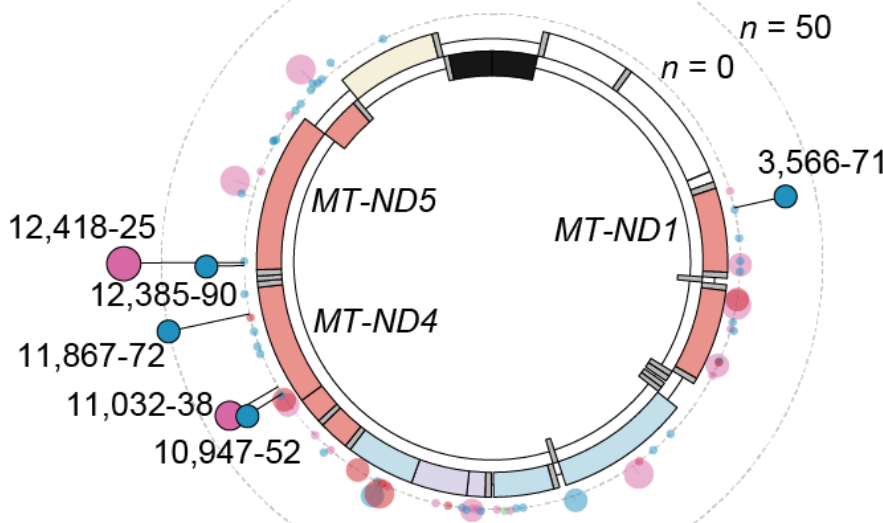

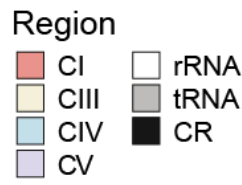

Nucleotide

- $A \bigcirc \mathrm{C} \odot \mathrm{G} \odot \mathrm{T}$

Repeat

length (bp)

$5 \mathrm{COO} 8$ 
743 Fig. 2: Truncating variants preferentially target complex I. a) Comparison of truncating

744 mutation rate (truncating variants/Mb) between OXPHOS complexes I, III, IV, V. Synonymous

745 mutation rates shown below for comparison. Truncating mutations $n=352$; synonymous $n=475$.

$746 P$-values from two-sided Poisson-exact. Single asterisk denotes $P<0.1$; double asterisk $P<0.01$;

747 n.s., not significant. b) Validation of analysis in a) using data from $n=1,951$ whole-genome

748 sequenced tumors from ICGC/PCAWG after removing samples also in TCGA. Truncating

749 mutations $n=198$; synonymous $n=263$. $P$-values and asterisks as in a). c) Distributions of

750 truncating and silent mutation heteroplasmy (estimated by variant allele frequency) among

751 variants in OXPHOS complex I, III, IV, or V. Difference in heteroplasmy between truncating and

752 silent mutations calculated by two-sided Wilcoxon rank sum test. $\mathrm{Cl}, P=1 \times 10^{-6}$, not significant for

753 other complexes. d) Percentage of tumors with truncating mtDNA variants per cancer type,

754 among well-covered samples. Right, number of well-covered samples per cancer type. e)

755 Percentage of samples per cancer type with truncating variants affecting OXPHOS complex I or

756 III-V. Asterisk indicates cancertypes with enriched truncating variants targeting $\mathrm{Cl}$ compared to

757 CIII-V, $Q<0.01$, two-sided McNemar's test. f) Circular mtDNA genome annotated with 73

758 homopolymer repeat loci $\geq 5 \mathrm{bp}$ in length. Dot height from the circular mtDNA genome indicates

759 the number of affected samples, dot color indicates the identity of the repeated nucleotide (A, C,

$760 \mathrm{G}, \mathrm{T})$, dot width indicates the length of the repeat region (5-8bp). Includes putatively somatic

761 truncating variants with tumor-only sequencing coverage. The 6 solid-color homopolymer loci

762 highlighted were found to be statistically enriched hotspots for frame-shift indels in tumors. g)

763 The 73 homopolymer repeat loci arranged by gene and repeat size. Dot width indicates -

$764 \log _{10}(Q$-value $)$ for enriched frame-shift indels in tumors. The 6 hotspot loci are labeled. 
a

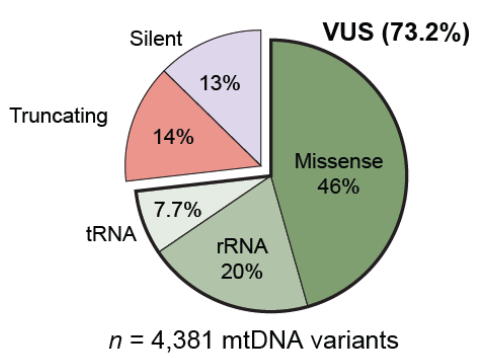

$\mathbf{e}$

$\mathbf{f}$
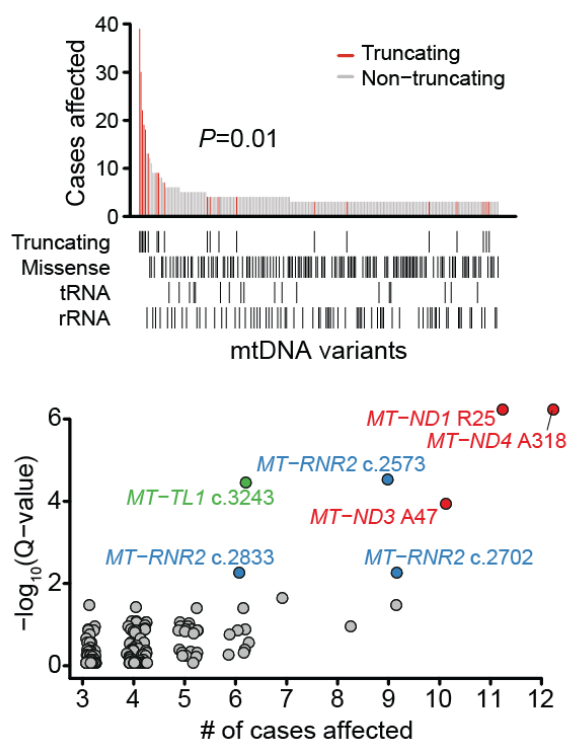

SNV Hotspots

- Protein-coding ○ rRNA ○ tRNA ○ Not significant
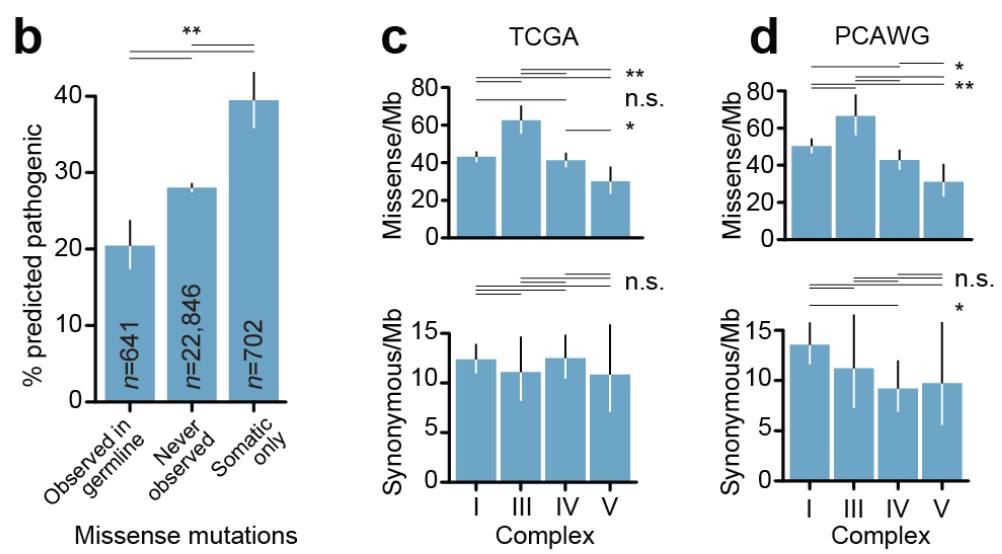

9

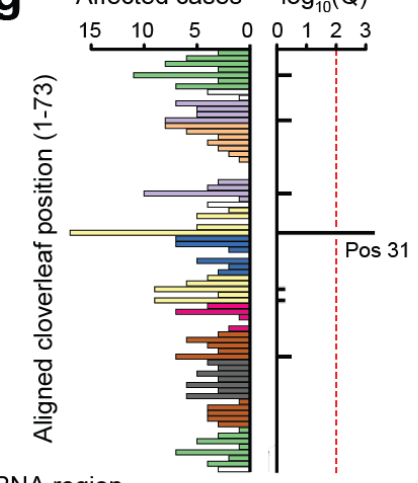

tRNA region

$\square$ Acceptor stem $\square$ Anti-codon stem $\square$ T-stem

$\square$ D-stem $\square$ Anti-codon loop $\square$ T-loop

$\square$ D-loop

$\square$ Variable region $\square$ n/a

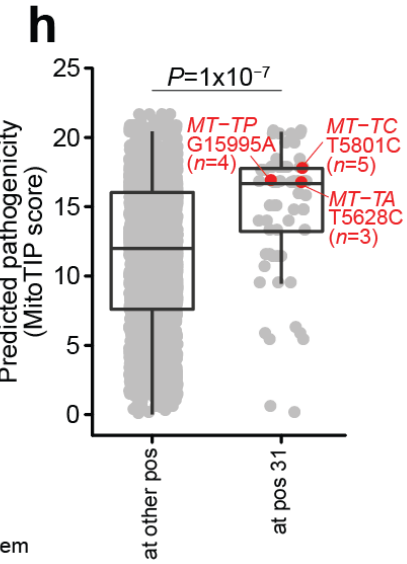

All tRNA mutations ... i

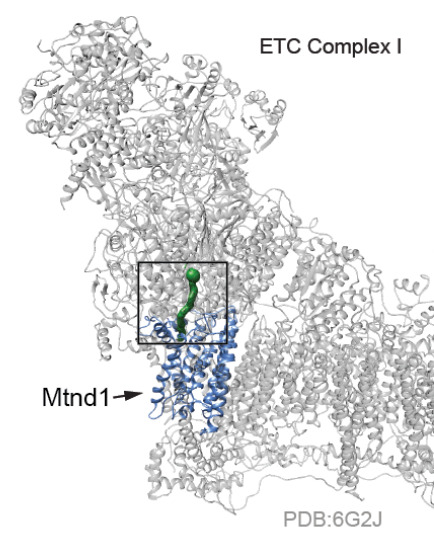

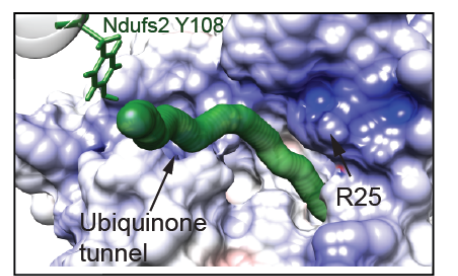

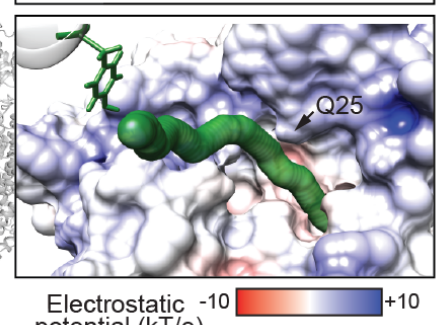

Electrostatic
potential (kT/e)

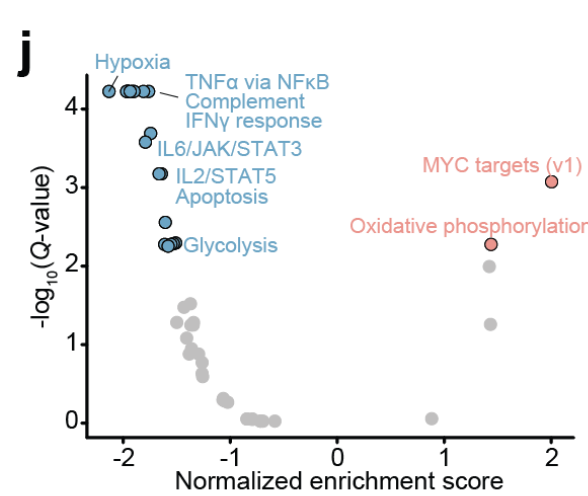

Change in expression (ND1 $1^{R 25}$ vs WT) O Up in MT-ND1 $1^{\text {R25Q }}$ O Down in MT-ND1 $1^{\text {R25Q }}$ n.s.

Fig. 3: Non-truncating mtDNA mutations arise as rare recurrent alleles in protein-coding and RNA elements. a) The proportion of truncating, synonymous and VUS somatic mtDNA mutations in this study. VUSs are further classified into missense protein-coding variants, or mutations to rRNA or tRNA genes. b) Comparison of the percentage of unique VUSs predicted to be pathogenic by APOGEE ${ }^{20}$ between somatic variants which (1) were ever observed to also 
772 arise as germline variants among $200 \mathrm{~K}$ normal samples from HelixMTdb; (2) were never

773 observed somatically mutated; or (3) were only observed as somatic mutations. All indicated

774 comparisons were statistically significant with $\mathrm{P}<10^{-4}$. c) Comparison of missense mutation rate

775 (missense variants/Mb) between OXPHOS complexes I, III, IV, V. Synonymous mutation rates

776 shown below for comparison. Missense mutations $n=1,718$; synonymous $n=475$. $P$-values from

777 two-sided Poisson-exact. Single asterisk denotes $P<0.1$; double asterisk $P<0.01$; n.s., not

778 significant. d) Validation of analysis in a) using data from $n=1,951$ whole-genome sequenced

779 tumors from ICGC/PCAWG after removing samples also in TCGA. Missense mutations $n=1073$;

780 synonymous $n=263$. $P$-values and asterisks as in a). e) Rare-recurrent alleles are primarily non-

781 truncating variants. Top portion, number of samples with the given mtDNA mutant allele in

782 decreasing order of prevalence (mutations called in samples with adequate tumor and normal

783 coverage). Bottom portion, tracks indicate consequence of corresponding variant in top portion.

784 f) Individual base-pair positions in mtDNA with somatic single-nucleotide variants (SNVs) in $\geq 5$

785 tumors, and their statistical enrichment for mutations. Hotspot positions with $Q<0.01$ are colored

786 by the type of gene in which they arise (protein-coding, rRNA or tRNA). Select hotspots are

787 labeled with their genomic positions (for mutations in tRNAs and rRNAs) or residue (protein

788 coding genes). g) Prevalence of SNVs in tRNA genes, aligned to their positions in the folded

789 tRNA cloverleaf structure. Bottom portion, number of samples with SNVs at the given tRNA

790 cloverleaf position across all tRNAs. Top portion, statistical enrichment for the aligned position

791 for mutations. h) Mutations at tRNA cloverleaf structural position 31 have greater predicted

792 pathogenicity scores (based on MitoTIP ${ }^{39}$ ) compared to all possible mutations at other

793 positions. tRNA mutations at position 31 affecting $\geq 2$ samples are highlighted. $P$-value from two-

794 sided Wilcoxon rank sum test. To reduce image size, a random selection of $5 \%$ of the mutations

795 not at position 31 are plotted ( $P$-value based on the complete set of mutations). i) The Mtnd1

$796 \mathrm{R} 25 \mathrm{Q}$ mutation lies at a critical region of complex I near the entrance to the ubiquinone binding

797 tunnel (dotted green path), likely affecting its capacity for binding ubiquinone. Larger view: The

798 complete mammalian complex I structure (gray) highlighting Mtnd1 (blue), and the ubiquinone

799 binding tunnel (green) and binding site (large green sphere); black box indicates the region in

800 the closeup view. Closeups, the predicted surface electrostatic potential of Mtnd1 (top) wild-type

801 and R25Q mutant (bottom), proximal to the ubiquinone binding tunnel (green), leading to its

802 binding site at Ndufs2 Y108. j) Differentially expressed mSigDB Hallmark genesets between

803 colorectal tumors with MT-ND1 R25Q and those without non-silent somatic mtDNA variants (i.e.

804 wild-type). Normalized enrichment score (NES) and adjusted P-values based on gene set

805 enrichment analysis (GSEA) using the fgsea R package ${ }^{44}$. 
b

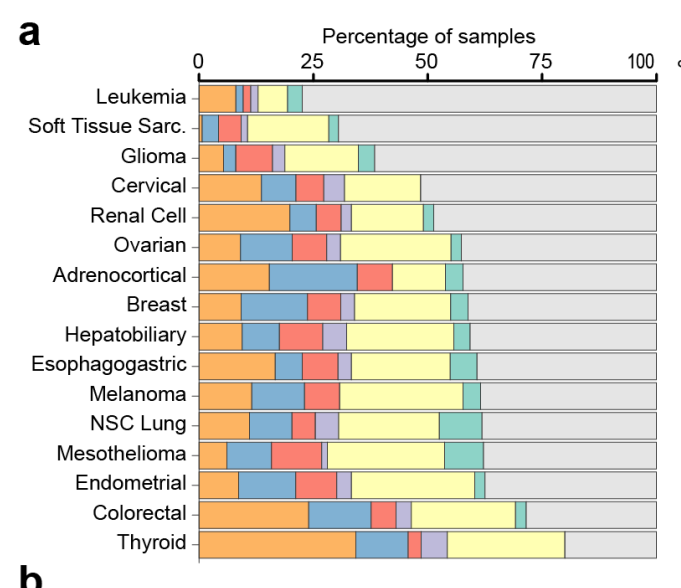

$n$ samples
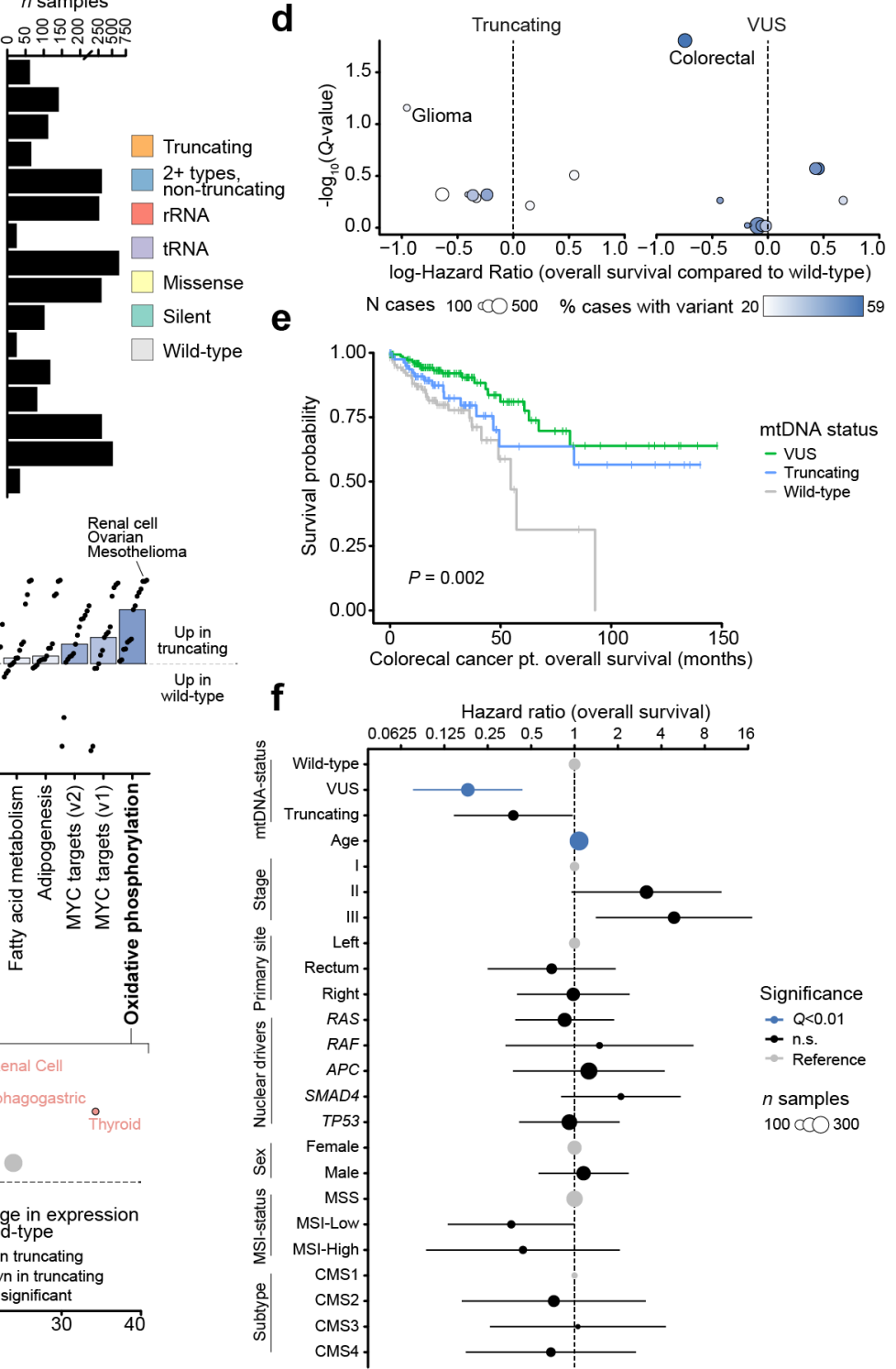

Fig. 4: Mitochondrial genotypes associate with transcriptional and clinical phenotypes. a)

Percentage of well-covered tumors with different types of somatic mtDNA variants per cancer type. Right, number of well-covered samples per cancer type. b) Differential expression of mSigDB Hallmarks genesets, between samples with truncating mtDNA variants and those with no nonsynonymous somatic mutations (i.e. "wild-type" samples). Differential expression is quantified by directional - $\log _{10}(Q$-value): greater than 0 denotes up-regulation in samples with truncating variants, below 0 denotes up-regulation in wild-type samples. Each dot is a single cancer type's level of dysregulation of that geneset. Bars show the median level of dysregulation across 15 cancer types; bar shading shows the number of cancer types with 
818 NFKB Signaling (left) and Oxidative Phosphorylation (right) genesets in individual cancer types.

819 X-axis shows the overall proportion of samples of each cancer type with truncating variants; Y-

820 axis matches the $\mathrm{Y}$-axis in $\mathrm{b}$ ). Dot width denotes number of well-covered samples for each

821 cancer type. d) Effect size and statistical significance of mtDNA truncating variants (left) and

822 VUSs (right) on overall survival among individual cancer types. Effect sizes (quantified as log-

823 hazard ratios) are from univariate Cox proportional-hazards models run for each cancer type

824 independently. $Q$-values are adjusted $P$-values from the model coefficients for each cancer

825 type.

826 e) Kaplan-Meier plot showing difference in overall survival time among $n=344$ TCGA colorectal

827 cancer patients with somatic VUSs $(n=152)$, truncating variants $(n=84)$, or no nonsynonymous

828 mutations (i.e. wild-type, $n=108$ ). f) Multivariate analysis of the effect of mtDNA variants on

829 overall survival time among $n=344$ TCGA colorectal cancer patients (stage 1-3). Truncating

830 variants and VUSs are each compared to wild-type samples, while controlling for known

831 prognostic clinical and genomic covariates using a Cox proportional-hazards model. Hazard

832 ratios for each covariate are shown on a log-scale, error-bars are 95\% confidence intervals from

833 the Cox proportional hazards regression. Point size indicates the number of samples with the

834 associated covariate value (except for Age, which was coded as a continuous variable, and

835 therefore the size corresponds to the total number of samples). Blue points are statistically

836 significant $(Q$-value $<0.01)$; black points not significant; gray points are reference categories

837 and were not tested.

\section{Supplementary Materials}

\section{Supplementary Tables}

846 Supplementary Table 1: Table of mtDNA position 1-16,569 annotated with gene symbols,

847 encoding strand, nucleotide, and exclusion criteria.

848 Supplementary Table 2: Table of mutation rates in mtDNA and nuclear cancer-associated 849 genes.

850 Supplementary Table 3: Table of SNV hotspot positions and associated significance and 851 annotations. 
Supplementary Table 4: Table of homopolymer indel hotspots and associated significance and

853 annotations.

854 Supplementary Table 5: Table of tRNA structural alignment hotspots and significance and 855 annotations.

856 Supplementary Table 6: Table with mtDNA variants and mtDNA status classifications for all 857 TCGA samples included in this study.

\section{Supplementary Figures}

861

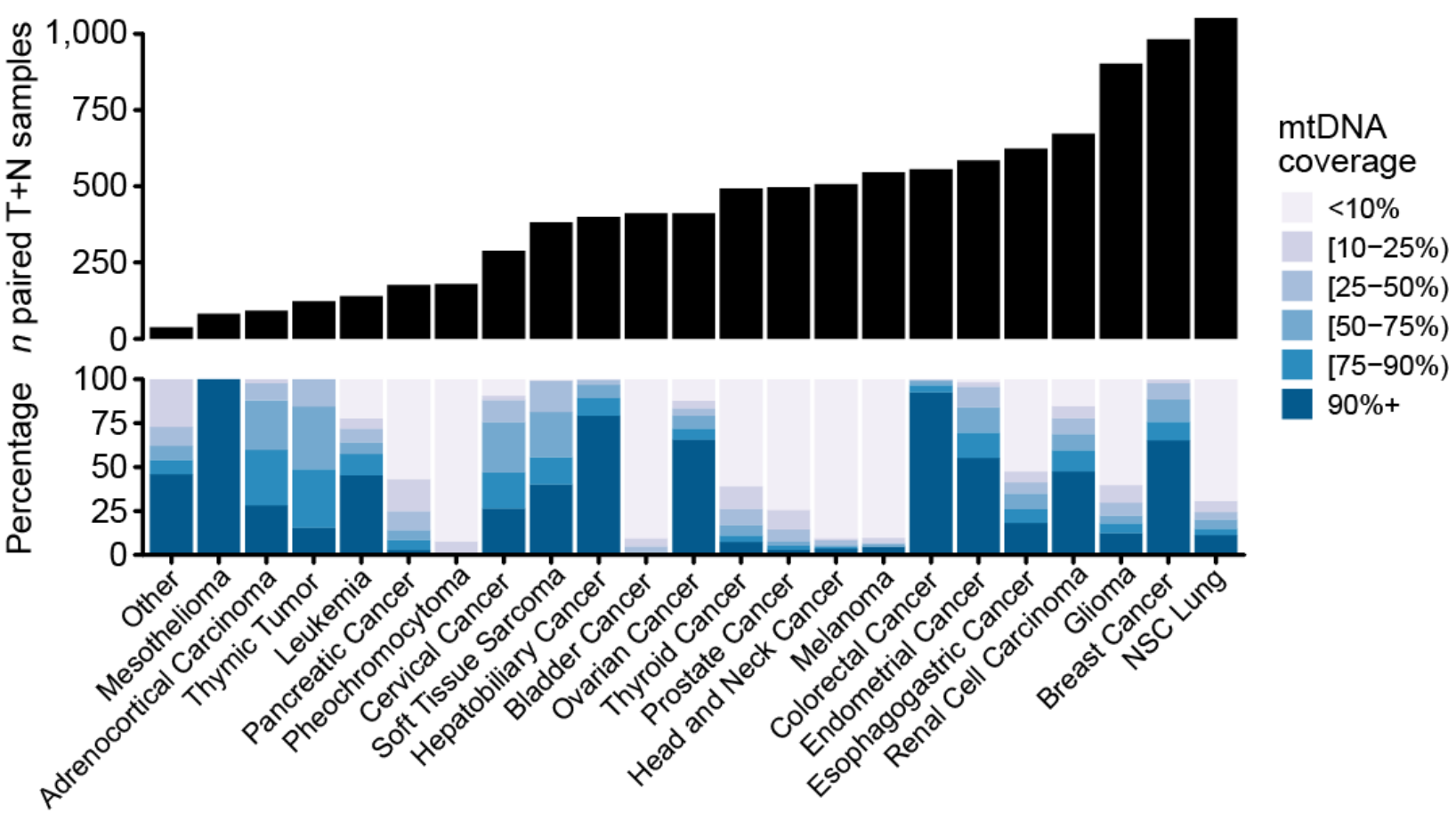

863 Supplementary Fig. 1a: Distribution of cancer types in patient cohort. Top, number of

864 tumor samples and paired matched normal samples per cancer type in this study. Bottom, the

865 proportion of tumor and normal sample pairs each with $\geq 5$ read coverage in the indicated

866 percentage of genic regions of the mitochondrial genome (e.g. darkest blue indicates the

867 percent of well-covered samples of the given cancer type.). 


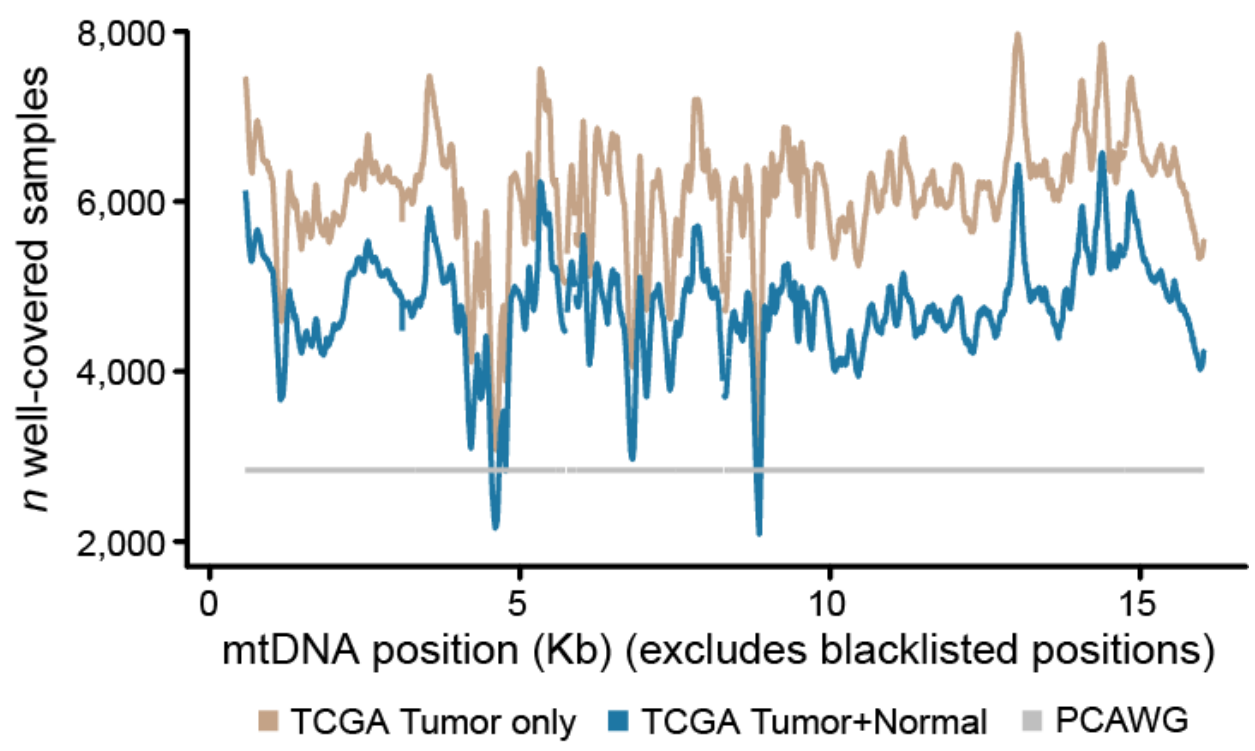
number of samples for which the given mtDNA position was sequenced to adequate depth to call somatic variants. Brown, the number of samples using unpaired tumor-only data, applicable only for protein-truncating variants which were always assumed to be of somatic origin. Blue,

874 the number using paired tumor and matched-normal data, applicable for all non-truncating 875 variants which required evidence that the variant was absent in the matched normal to be confidently classified as of somatic origin. Gray, the number of whole-genome sequenced samples available from ICGC/PCAWG for comparison.

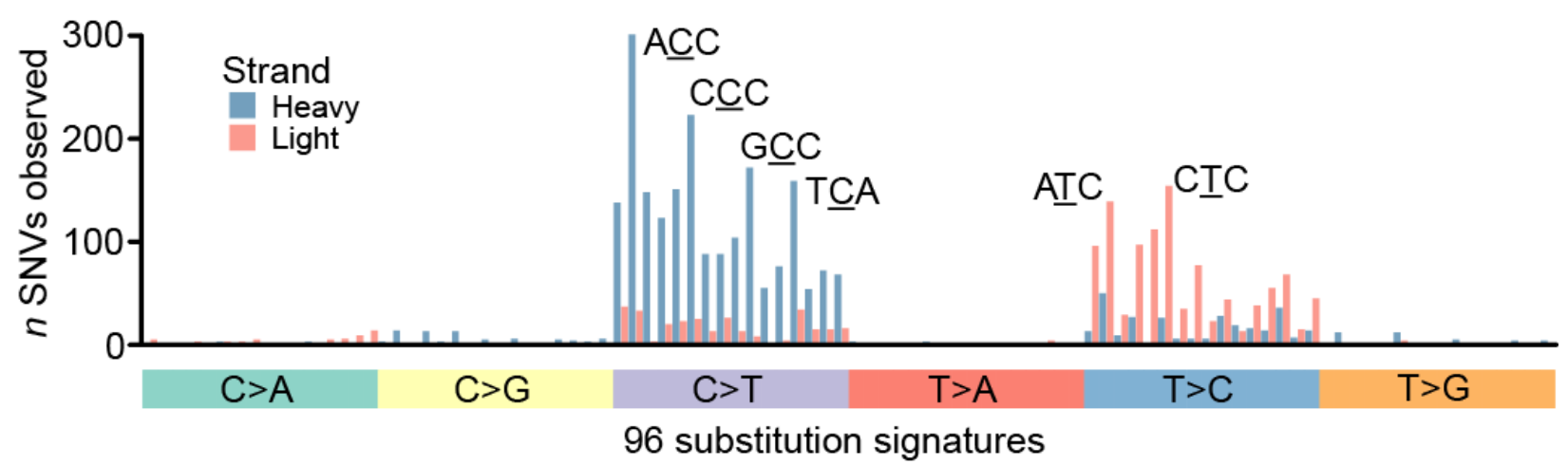

882 Supplementary Fig. 1c: Strand-specific mutational signatures in our dataset. The

883 frequency of somatic SNVs on the light or heavy mtDNA strand with each of the 96 possible 884 mutational signatures with trinucleotide contexts (among $n=3,872 \mathrm{SNVs}$ ). Blue bars indicate 885 the prevalence of mutational signatures for heavy-strand encoded SNVs (substitutions at C or T 
central nucleotides); red bars indicate those for light-strand encoded SNVs (substitutions at G or

887 T nucleotides, which were standardized to their $\mathrm{C}$ or $\mathrm{T}$ complementary nucleotide). The most

888 prevalent mutational signatures are labeled. The underlined central position is mutated with the

889 single nucleotide substitution labeled in the tile below.

890
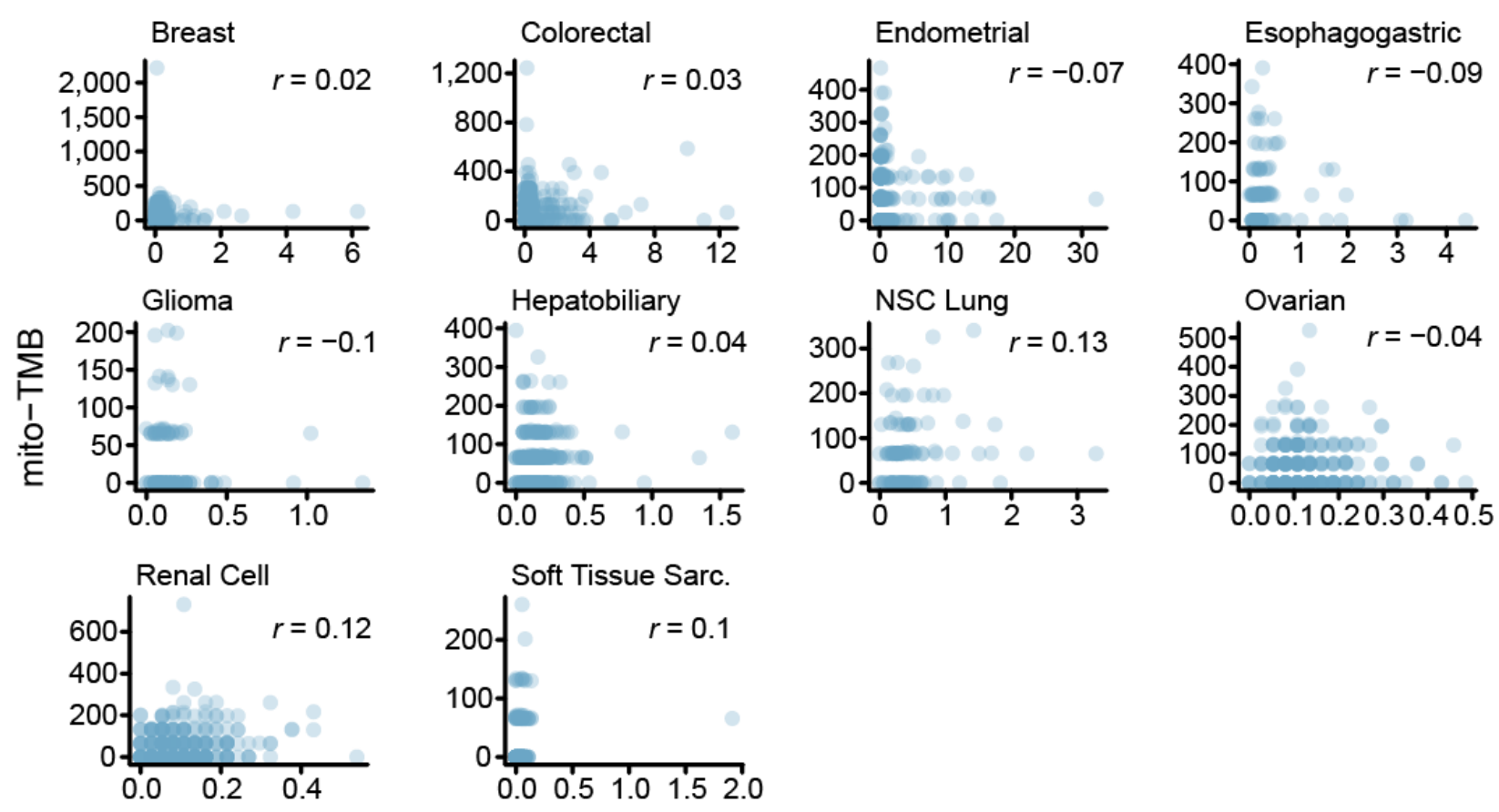

891

Nuclear TMB

892 Supplementary Fig. 1d: mtDNA mutation burden does not correlate with nuclear mutation

893 burden within cancer types. Mitochondrial and nuclear tumor mutation burdens (TMB,

894 mutations/Mb) are shown for each well-covered tumor, among cancer types with $n \geq 100$

895 samples. Nuclear TMBs are calculated based on mutations to 468 cancer-associated genes and 896 their total coding-sequence length. Pearson correlation coefficients $r$ indicate no linear correlation between mitochondrial and nuclear TMBs were observed for any cancer type tested. 


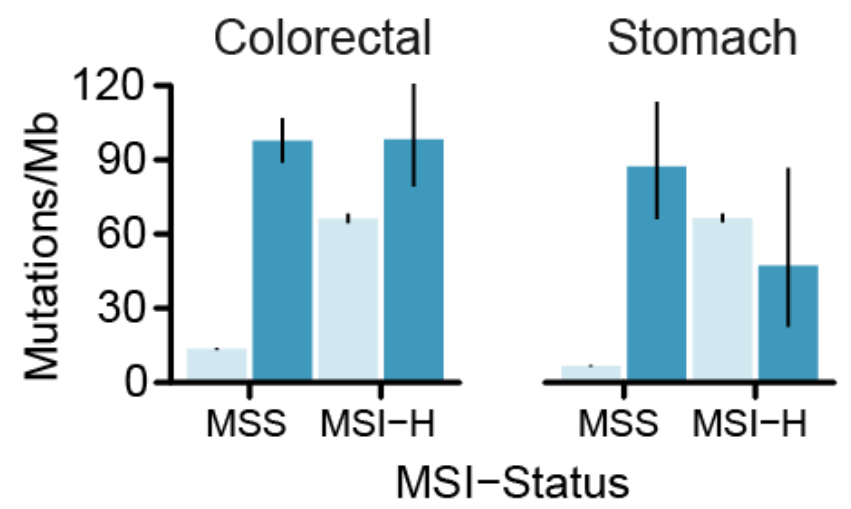

901 Supplementary Fig. 1e: Microsatellite instability does not affect somatic mtDNA mutation

902 rate. TMBs for somatic mtDNA mutations and mutations to cancer-associated genes are

903 compared between microsatellite stable (MSS) and microsatellite unstable (MSI-High) tumors,

904 for both ( $n$ colorectal cancer: $\mathrm{MSI}=65$, MSS=318; $n$ stomach adenocarcinomas: $\mathrm{MSI}=75$,

905 MSS=256). Although MSI-High tumors have elevated TMB for nuclear cancer genes, there is no

906 effect on mtDNA TMB. Moreover, mtDNA TMB is similar to (or exceeds) that of nuclear cancer

907 associated genes in both cancer types. Error bars are 95\% Poisson exact confidence intervals.

908

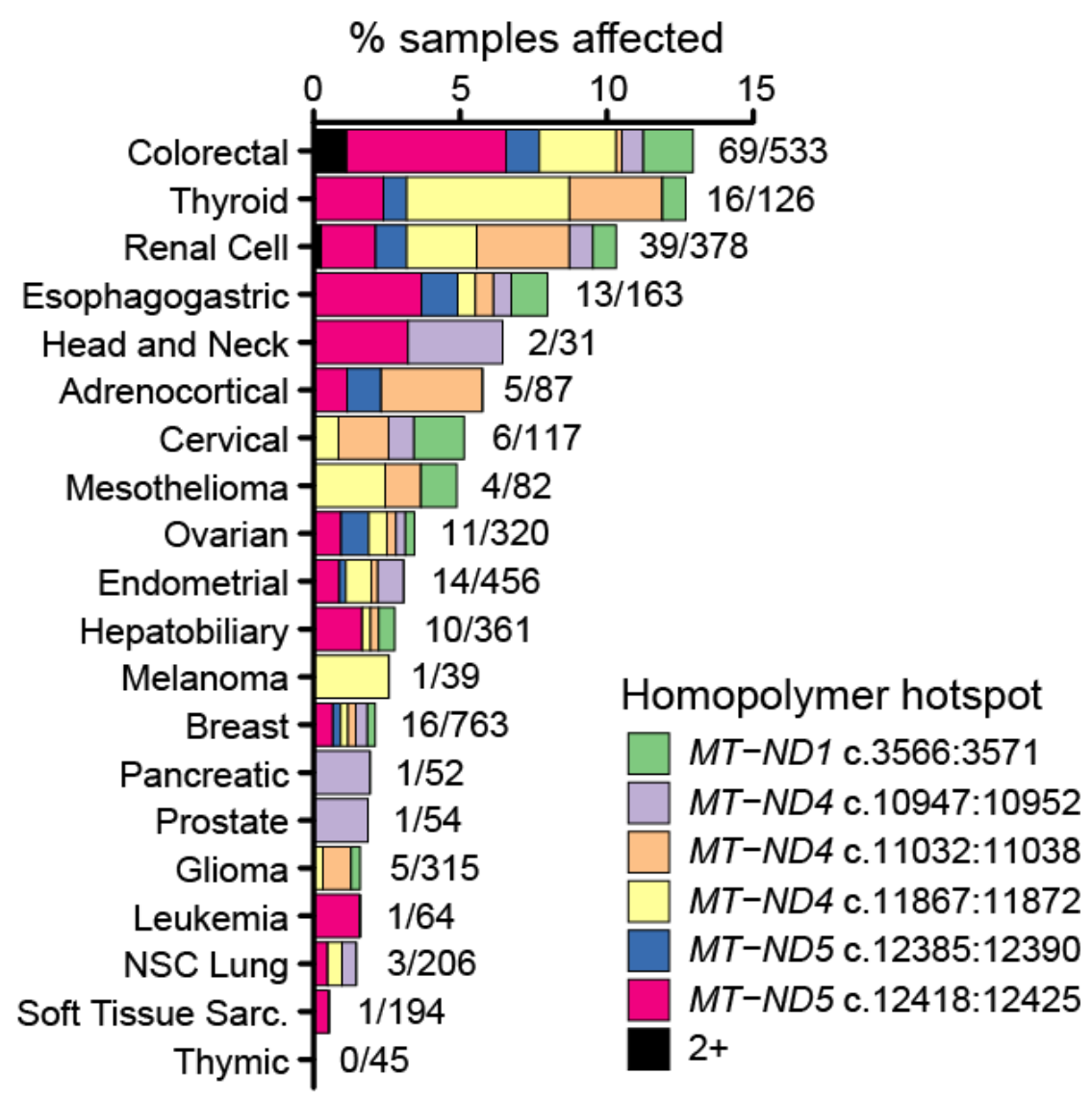


910 Supplementary Fig. 2a: Prevalence of frame-shift indels at homopolymer hotspots across

911 cancer types. Percentage of cases per cancer type with truncating frame-shift indels at any of 6

912 indel hotspot loci. Plotted cancer types had $\geq 20$ well-covered samples ( $n=4,432$ paired tumor

913 and matched-normal samples total). Labels indicate the fraction of samples with any indels at

914 homopolymer hotspot out of the total number of well-covered samples for the given cancer type.

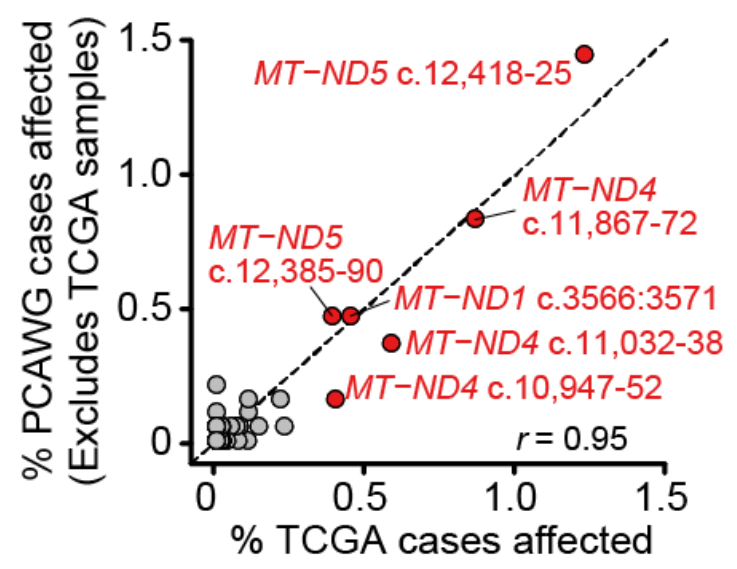

Homopolymer hotspot

918 Supplementary Fig. 2b: Validation of homopolymeric indel hotspot loci. The proportion of

919 samples in TCGA (X-axis) or PCAWG (excluding samples also in TCGA, Y-axis) with frame-

920 shift indels at 73 homopolymeric regions. The 6 indel hotspot loci are colored red and labeled.

$921 \mathrm{y}=\mathrm{x}$ is shown as a dashed line. Pearson correlation coefficient $r$ as indicated. 


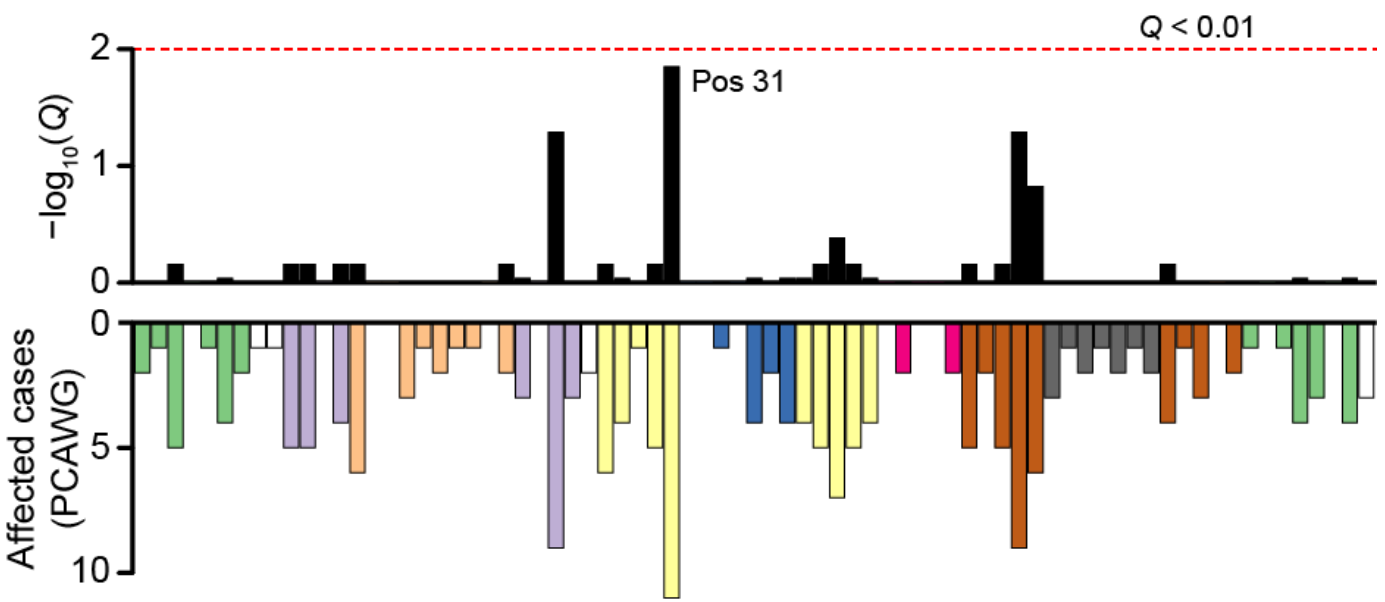

Aligned cloverleaf position (1-73)

Supplementary Fig. 3c: Validation of tRNA structural hotspots in PCAWG. The number of samples with SNVs in tRNAs at the indicated cloverleaf structural position, bottom; top, the statistical enriched of the given position for mutations. Position $31 \mathrm{Q}$-value=0.014, $n=196$ tRNA mutations among 1,951 PCAWG samples.

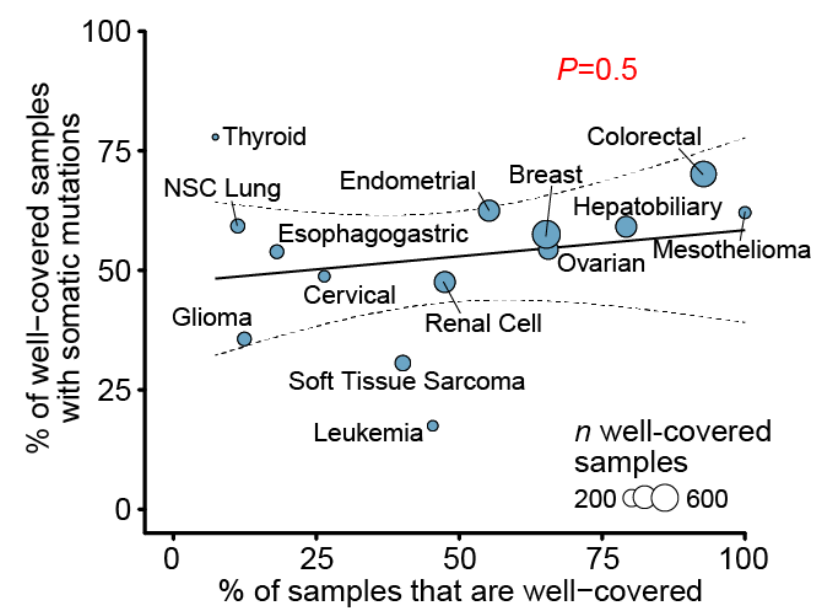

930 Supplementary Fig. 4a: Proportion of samples with detectable mutations is not biased by

931 cancer type sequencing coverage. There is no correlation between the fraction of well-

932 covered samples in a cancer type and the proportion of well-covered samples with a detectable

933 somatic mtDNA mutation. Cancer types with $\geq 30$ well-covered samples shown, $P$ value from

934 linear regression. 


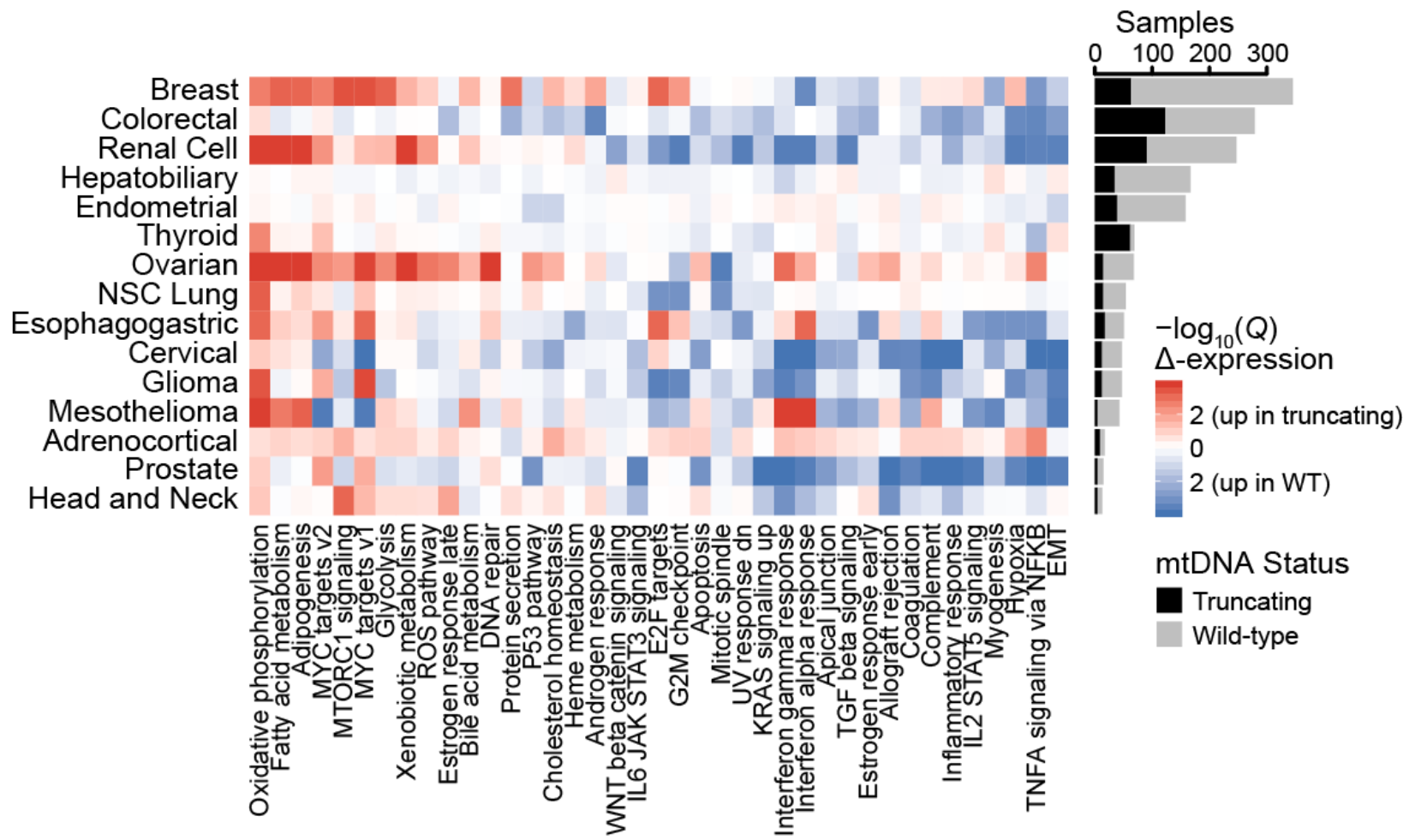

937 variants. (Left) Heatmap shows directional significance of dysregulation of a given geneset in 938 tumors with truncating variants among the given cancer type; - $\log _{10}(Q$-value $)>2$ indicates 939 significant up-regulation, <-2 indicates significant down-regulation. (Right) Histogram of wild-

940 type samples and samples with truncating variants used to calculate differentially-expressed

941 genes and dysregulated genesets. 


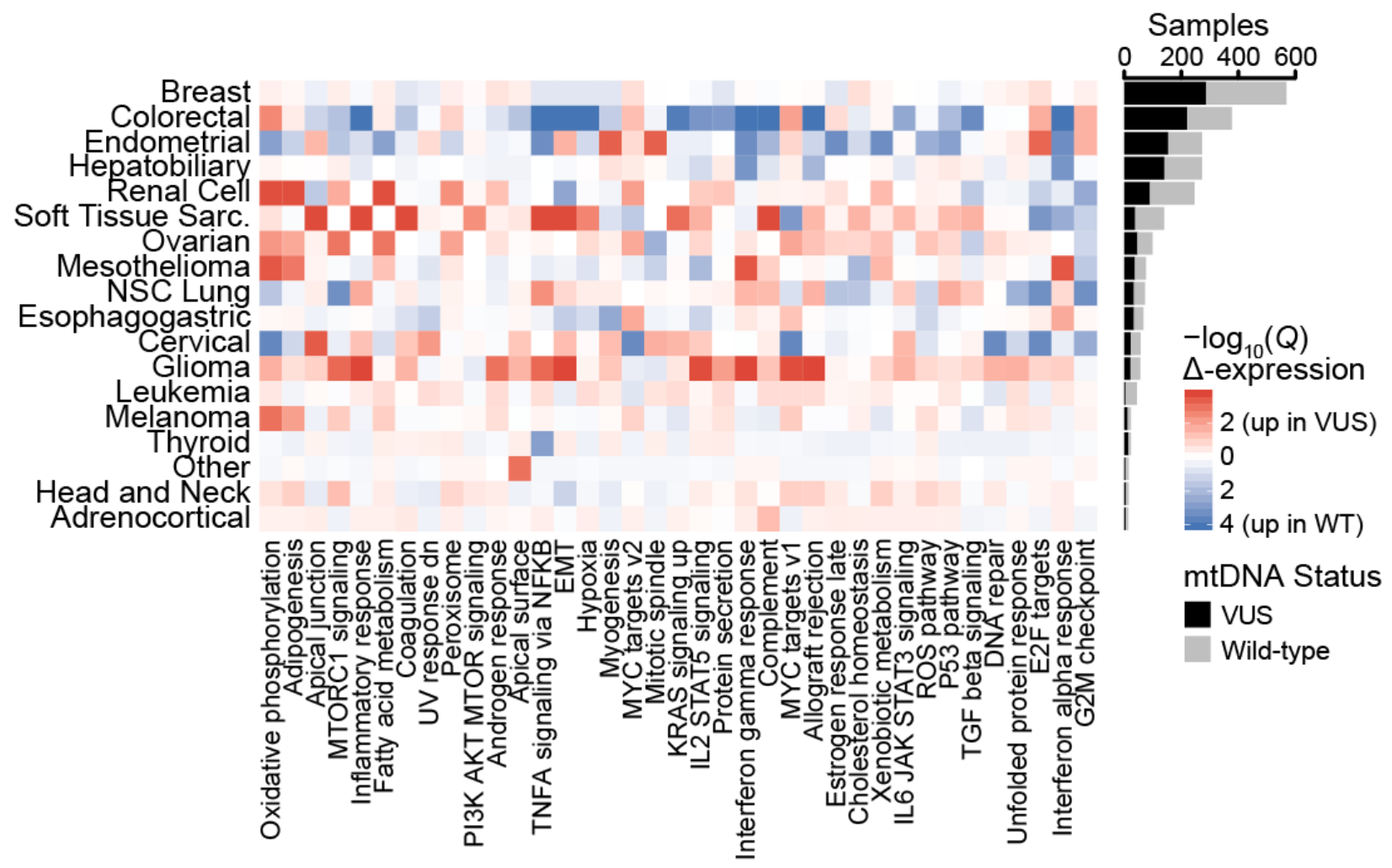

Supplementary Fig. 4c: Transcriptional dysregulation attributed to mtDNA VUSs.

Heatmap, differentially expressed mSigDB Hallmarks genesets between tumors with any somatic VUSs or wild-type mtDNA. Genesets ordered from most up-regulated across cancer types to most down-regulated. Barplot, number of cases with VUSs or wild-type mtDNA.

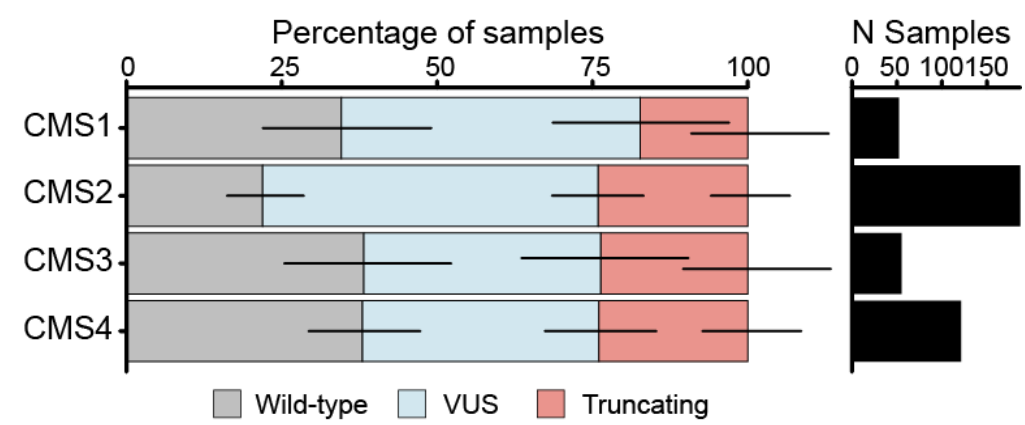

\section{Supplementary Fig. 4d: Difference in mtDNA mutation status between colorectal cancer} consensus molecular subtypes. Left, the proportion of samples with wild-type mtDNA (i.e. no somatic mutations), VUS (any non-truncating) or truncating variants among colorectal tumors with each consensus molecular subtype (CMS) is shown. Right, histogram of the number of well-covered colorectal tumors. There was a statistically significant difference in mtDNA 
mutation status between different CMS classifications $(P=0.03$, Chi-squared test, $n=415$

957 samples total).

958

959

960 Bibliography

961 1. Rheinbay, E. et al. Analyses of non-coding somatic drivers in 2,658 cancer whole genomes.

$962 \quad$ Nature 578, 102-111 (2020).

963 2. Ju, Y. S. et al. Origins and functional consequences of somatic mitochondrial DNA

964 mutations in human cancer. elife 3, (2014).

965 3. Yuan, Y. et al. Comprehensive molecular characterization of mitochondrial genomes in

966 human cancers. Nat. Genet. 52, 342-352 (2020).

967 4. Stewart, J. B. et al. Simultaneous DNA and RNA Mapping of Somatic Mitochondrial Mutations across Diverse Human Cancers. PLoS Genet. 11, e1005333 (2015).

969 5. Grandhi, S. et al. Heteroplasmic shifts in tumor mitochondrial genomes reveal tissuespecific signals of relaxed and positive selection. Hum. Mol. Genet. 26, 2912-2922 (2017).

971 6. Hopkins, J. F. et al. Mitochondrial mutations drive prostate cancer aggression. Nat.

$972 \quad$ Commun. 8, 656 (2017).

973 7. To, T.-L. et al. A Compendium of Genetic Modifiers of Mitochondrial Dysfunction Reveals 974 Intra-organelle Buffering. Cell 179, 1222-1238.e17 (2019).

975 8. Birsoy, K. et al. An essential role of the mitochondrial electron transport chain in cell 976 proliferation is to enable aspartate synthesis. Cell 162, 540-551 (2015).

977 9. Samuels, D. C. et al. Finding the lost treasures in exome sequencing data. Trends Genet. $978 \quad 29,593-599(2013)$.

979 10. Cibulskis, K. et al. Sensitive detection of somatic point mutations in impure and 980 heterogeneous cancer samples. Nat. Biotechnol. 31, 213-219 (2013).

981 11. Li, H. et al. The Sequence Alignment/Map format and SAMtools. Bioinformatics 25, 2078- 
2079 (2009).

983 12. Collura, R. V., Auerbach, M. R. \& Stewart, C. B. A quick, direct method that can

984 differentiate expressed mitochondrial genes from their nuclear pseudogenes. Curr. Biol. 6, $985 \quad 1337-1339(1996)$.

986 13. Cheng, D. T. et al. Memorial Sloan Kettering-Integrated Mutation Profiling of Actionable 987 Cancer Targets (MSK-IMPACT): A Hybridization Capture-Based Next-Generation Sequencing Clinical Assay for Solid Tumor Molecular Oncology. J. Mol. Diagn. 17, 251-264

14. Bolze, A. et al. Selective constraints and pathogenicity of mitochondrial DNA variants inferred from a novel database of 196,554 unrelated individuals. BioRxiv (2019). doi:10.1101/798264

15. Chang, M. T. et al. Identifying recurrent mutations in cancer reveals widespread lineage diversity and mutational specificity. Nat. Biotechnol. 34, 155-163 (2016).

16. Chang, M. T. et al. Accelerating discovery of functional mutant alleles in cancer. Cancer Discov. 8, 174-183 (2018).

997 17. Gopal, R. K. et al. Early loss of mitochondrial complex I and rewiring of glutathione

18. Triska, P. et al. Landscape of germline and somatic mitochondrial DNA mutations in pediatric malignancies. Cancer Res. 79, 1318-1330 (2019).

19. Alston, C. L. et al. A novel mitochondrial MTND5 frameshift mutation causing isolated complex I deficiency, renal failure and myopathy. Neuromuscul. Disord. 20, 131-135

1004 20. Castellana, S. et al. High-confidence assessment of functional impact of human 1005 mitochondrial non-synonymous genome variations by APOGEE. PLoS Comput. Biol. 13, $1006 \quad$ e1005628 (2017).

1007 21. Martínez-Reyes, I. et al. Mitochondrial ubiquinol oxidation is necessary for tumour growth. 
Nature (2020). doi:10.1038/s41586-020-2475-6

1009

1010

1011

1012

1013

1014

1015

1016

1017

1018

1019

1020

1021

1022

1023

1024

1025

1026

1027

1028

1029

1030

1031

1032

1033

22. El-Hattab, A. W., Adesina, A. M., Jones, J. \& Scaglia, F. MELAS syndrome: Clinical manifestations, pathogenesis, and treatment options. Mol. Genet. Metab. 116, 4-12 (2015).

23. Gorman, G. S. et al. Prevalence of nuclear and mitochondrial DNA mutations related to adult mitochondrial disease. Ann. Neurol. 77, 753-759 (2015).

24. Gopal, R. K. et al. Widespread chromosomal losses and mitochondrial DNA alterations as genetic drivers in hürthle cell carcinoma. Cancer Cell 34, 242-255.e5 (2018).

25. Spagnolo, M. et al. A new mutation in the mitochondrial tRNA(Ala) gene in a patient with ophthalmoplegia and dysphagia. Neuromuscul. Disord. 11, 481-484 (2001).

26. Miller, M. L. et al. Pan-Cancer Analysis of Mutation Hotspots in Protein Domains. Cell Syst. 1, 197-209 (2015).

27. Gao, J. et al. 3D clusters of somatic mutations in cancer reveal numerous rare mutations as functional targets. Genome Med. 9, 4 (2017).

28. Horváth, R., Reilmann, R., Holinski-Feder, E., Ringelstein, E. B. \& Klopstock, T. The role of complex I genes in MELAS: a novel heteroplasmic mutation 3380G>A in ND1 of mtDNA. Neuromuscul. Disord. 18, 553-556 (2008).

29. Agip, A.-N. A. et al. Cryo-EM structures of complex I from mouse heart mitochondria in two biochemically defined states. Nat. Struct. Mol. Biol. 25, 548-556 (2018).

30. Joshi, S. et al. The genomic landscape of renal oncocytoma identifies a metabolic barrier to tumorigenesis. Cell Rep. 13, 1895-1908 (2015).

31. Ganly, I. et al. Integrated genomic analysis of hürthle cell cancer reveals oncogenic drivers, recurrent mitochondrial mutations, and unique chromosomal landscapes. Cancer Cell 34, 256-270.e5 (2018).

32. Guinney, J. et al. The consensus molecular subtypes of colorectal cancer. Nat. Med. 21, 1350-1356 (2015).

33. Yaeger, R. et al. Clinical sequencing defines the genomic landscape of metastatic 
colorectal cancer. Cancer Cell 33, 125-136.e3 (2018).

1035

1036

1037

1038

1039

1040

1041

1042

1043

1044

1045

1046

1047

1048

1049

1050

1051

1052

1053

1054

1055

1056

1057

1058

1059

34. Priolo, C. et al. Impairment of gamma-glutamyl transferase 1 activity in the metabolic pathogenesis of chromophobe renal cell carcinoma. Proc Natl Acad Sci USA 115, E6274E6282 (2018).

35. Cancer Genome Atlas Research Network et al. The Cancer Genome Atlas Pan-Cancer analysis project. Nat. Genet. 45, 1113-1120 (2013).

36. McKenna, A. et al. The Genome Analysis Toolkit: a MapReduce framework for analyzing next-generation DNA sequencing data. Genome Res. 20, 1297-1303 (2010).

37. Li, H. A statistical framework for SNP calling, mutation discovery, association mapping and population genetical parameter estimation from sequencing data. Bioinformatics 27, 29872993 (2011).

38. Chakravarty, D. et al. Oncokb: A precision oncology knowledge base. JCO Precis. Oncol. 2017, (2017).

39. Sonney, S. et al. Predicting the pathogenicity of novel variants in mitochondrial tRNA with MitoTIP. PLoS Comput. Biol. 13, e1005867 (2017).

40. Love, M. I., Huber, W. \& Anders, S. Moderated estimation of fold change and dispersion for RNA-seq data with DESeq2. Genome Biol. 15, 550 (2014).

41. Zhu, A., Ibrahim, J. G. \& Love, M. I. Heavy-tailed prior distributions for sequence count data: removing the noise and preserving large differences. Bioinformatics 35, 2084-2092 (2019).

42. Benjamini, y Y. et al. Controlling the False Discovery Rate : A Practical and Powerful Approach to Multiple Testing Author ( $s$ ): Yoav Benjamini and Yosef Hochberg Source : Journal of the Royal Statistical Society . Series B ( Methodological ), Vol . 57 , No . 1 Published by : J R Statist Soc B 57, 289-300 (1995).

43. Liberzon, A. et al. The Molecular Signatures Database (MSigDB) hallmark gene set collection. Cell Syst. 1, 417-425 (2015). 
44. Sergushichev, A. An algorithm for fast preranked gene set enrichment analysis using cumulative statistic calculation. BioRxiv (2016). doi:10.1101/060012

45. Liu, Y. et al. Comparative molecular analysis of gastrointestinal adenocarcinomas. Cancer Cell 33, 721-735.e8 (2018).

1064 46. Ellrott, K. et al. Scalable open science approach for mutation calling of tumor exomes using multiple genomic pipelines. Cell Syst. 6, 271-281.e7 (2018).

1066 47. Pettersen, E. F. et al. UCSF Chimera-a visualization system for exploratory research and 1067 analysis. J. Comput. Chem. 25, 1605-1612 (2004).

1068 48. Jurcik, A. et al. CAVER Analyst 2.0: analysis and visualization of channels and tunnels in protein structures and molecular dynamics trajectories. Bioinformatics 34, 3586-3588 (2018).

49. Zhu, J., Vinothkumar, K. R. \& Hirst, J. Structure of mammalian respiratory complex I.

1073 50. Baker, N. A., Sept, D., Joseph, S., Holst, M. J. \& McCammon, J. A. Electrostatics of nanosystems: application to microtubules and the ribosome. Proc Natl Acad Sci USA 98,

1076 51. Dolinsky, T. J., Nielsen, J. E., McCammon, J. A. \& Baker, N. A. PDB2PQR: an automated 1077 pipeline for the setup of Poisson-Boltzmann electrostatics calculations. Nucleic Acids Res. 32, W665-7 (2004). 